Portland State University

PDXScholar

Fall 12-21-2015

\title{
Analysis of Capillary Flow in Interior Corners : Perturbed Power Law Similarity Solutions
}

Joshua Thomas McCraney

Portland State University

Follow this and additional works at: https://pdxscholar.library.pdx.edu/open_access_etds

Part of the Fluid Dynamics Commons, and the Mechanical Engineering Commons Let us know how access to this document benefits you.

Recommended Citation

McCraney, Joshua Thomas, "Analysis of Capillary Flow in Interior Corners : Perturbed Power Law Similarity Solutions" (2015). Dissertations and Theses. Paper 2725.

https://doi.org/10.15760/etd.2721

This Thesis is brought to you for free and open access. It has been accepted for inclusion in Dissertations and Theses by an authorized administrator of PDXScholar. Please contact us if we can make this document more accessible: pdxscholar@pdx.edu. 
Analysis of Capillary Flow in Interior Corners: Perturbed Power Law Similarity Solutions

\title{
by
}

Joshua Thomas McCraney

\begin{abstract}
A thesis submitted in partial fulfillment of the requirements for the degree of
\end{abstract}

\author{
Master of Science \\ in \\ Mechanical Engineering
}

Thesis Committee:

Mark Weislogel, Chair

Gerald Recktenwald

Derek Tretheway

Portland State University

2015 
(c) 2015 Joshua Thomas McCraney 


\begin{abstract}
The design of fluid management systems requires accurate models for fluid transport. In the low gravity environment of space, gravity no longer dominates fluid displacement; instead capillary forces often govern flow. This thesis considers the redistribution of fluid along an interior corner. Following a rapid reduction of gravity, fluid advances along the corner measured by the column length $z=\mathcal{L}(t)$, which is governed by a nonlinear partial differential equation with dynamical boundary conditions. Three flow types are examined: capillary rise, spreading drop, and tapered corner. The spreading drop regime is shown to exhibit column length growth $\mathcal{L} \sim t^{2 / 5}$, where a closed form analytic solution exists. No analytic solution is available for the capillary rise problem. However, a perturbed power law similarity solution is pursued to approximate an analytic solution in the near neighborhood of the exact solution for the spreading drop. It is recovered that $\mathcal{L} \sim t^{1 / 2}$ for the capillary rise problem. The tapered corner problem is not analytically understood and hence its corresponding $\mathcal{L}$ is undocumented. Based on the slender corner geometry, it is natural to hypothesize the tapered corner column length initially behaves like the capillary rise regime, but after sufficient time has elapsed, it transitions into the spreading drop regime. This leads to a conjecture that its column length growth $\mathcal{L}$ is restricted to $t^{2 / 5} \lesssim \mathcal{L} \lesssim t^{1 / 2}$. To verify this conjecture an explicit finite difference numerical solution is developed for all three regimes. As will be shown, the finite difference scheme converges towards the analytic solutions for the spreading drop and capillary rise regimes. From this we assume the finite difference scheme is accurate for corner flows of similar geometries,
\end{abstract}


and thus apply this scheme the more onerous criteria of the tapered corner. Numerical results support the conjectured $\mathcal{L}$ behavior for the tapered corner. Understanding the dynamics of such flows and responses to various geometries offers design advantages for spacecraft waste-management systems, fuel control, hydration containment, cryogenic flows, and a myriad of other fluid applications. 


\section{Acknowledgements}

I am grateful for the support of Fariborz Maseeh and the Masseeh Fellowship and the NASA Cooperative Agreement NNX12A047A.

I would like to thank Mark Weislogel for sound expertise and guidance concerning the entire scope of this work. Few professors offer the direction you do; thank you.

Much thanks to Gerry Recktenwald for streamlining code and offering technical suggestions.

And lastly, thank you Grandma Jo, whose prayers and encouragement played a paramount role in the completion of this work. I love you so much. 


\section{Contents}

Abstract $\quad$ i

Acknowledgements $\quad$ iii

List of Figures $\quad$ vi

List of Tables $\quad$ vii

List of Symbols $\quad$ vii

1 Introduction and Motivation $\quad 1$

1.1 Background Research . . . . . . . . . . . . . . . . . . 1

1.2 Present Scope . . . . . . . . . . . . . . . . . . . . 2

2 Derivation of Governing Equation 5

3 Analytics 10

3.1 Similarity Transform . . . . . . . . . . . . . . . . . . . 10

3.1.1 Capillary Rise (Constant Height) . . . . . . . . . . . . . . . . 12

3.1.2 Spreading Drop (Constant Volume) . . . . . . . . . . . . . . 13

3.1.3 Universal Similarity . . . . . . . . . . . . . . . . . 15

3.2 Analytical Solutions and Approximations . . . . . . . . . . . 16

3.2.1 The Perturbational Technique . . . . . . . . . . . . . 16

3.3 Variational Methods . . . . . . . . . . . . . . . . 24

3.4 Summary . . . . . . . . . . . . . . . . . . . 27

4 Numerical Analysis and Results 28

4.1 Generalized Finite Difference Scheme . . . . . . . . . . . . . . . . . 28

4.1.1 Capillary Rise (Constant Height) . . . . . . . . . . . . . . . . 35

4.1.2 Spreading Drop (Constant Volume) . . . . . . . . . . . . . . . 39

4.1.3 Tapered Corner . . . . . . . . . . . . . . . . . 46

4.2 Stability . . . . . . . . . . . . . . . 56

5 Conclusion $\quad 58$

5.1 Recommendations for Future Work . . . . . . . . . . . . . . 59

$\begin{array}{ll}\text { References } & 60\end{array}$ 
$\begin{array}{lll}\text { Appendix A Determination of } a \text { for Spreading Drop } & 62\end{array}$

$\begin{array}{lll}\text { Appendix B Volume Integral } & 64\end{array}$

$\begin{array}{ll}\text { Appendix C Volume Conservation and } \epsilon / F_{A} & 66\end{array}$

$\begin{array}{ll}\text { Appendix D Error Measure } & 68\end{array}$

$\begin{array}{lll}\text { Appendix E MATLAB Code Capillary Rise } & 69\end{array}$

$\begin{array}{lll}\text { Appendix F } & \text { MATLAB Code Spreading Drop } & 71\end{array}$

$\begin{array}{ll}\text { Appendix G MATLAB Code Tapered Corner } & 73\end{array}$ 


\section{List of Figures}

1.1 A sketch of the hypothesized behavior for the tapered corner $\mathcal{L} \ldots .3$

1.2 Illustration of the tapered corner regime . . . . . . . . . 4

2.1 Sketch of capillary rise and cross-section in an interior corner . . . . 6

3.1 The naive expansion (3.17) is plotted through various orders of $\varepsilon \ldots 21$

3.2 Maximum error . . . . . . . . . . . . . . . . . . . . . 22

$3.3 \phi\left(\eta^{+}\right)$is plotted plotted against a numerical solution $\ldots \ldots \ldots . .26$

4.1 Numerical results plotted from the capillary rise regime. . . . . . . . 37

4.2 Numerical results plotted from the capillary rise regime . . . . . . . . 37

4.3 Numerical results plotted from the capillary rise regime . . . . . . . . 38

4.4 Numerical results plotted from the spreading drop regime . . . . . . . 44

4.5 Numerical results plotted from the spreading drop regime . . . . . . . 44

4.6 Numerical results plotted from the spreading drop regime . . . . . . . 45

4.7 The first step in the finite difference scheme of capillary rise in a tapered corner flow . . . . . . . . . . . . . . . . . . . . 51

4.8 The second step in the finite difference scheme of capillary rise in a tapered corner flow . . . . . . . . . . . . . . . . 51

4.9 Numerical results for the tapered corner regime . . . . . . . . . . 54

4.10 Numerical results plotted from the tapered corner regime . . . . . . 55 


\section{List of Tables}

2.1 Non-dimensional independent and dependent variables . . . . . . . . 5

3.1 Summarized values from the similarity solutions . . . . . . . . . . . 13

3.2 Capillary rise analytically approximated data . . . . . . . . . . . . 23

4.1 Capillary rise numerical data . . . . . . . . . . . . . . . . . . . . . . . . . . . . . . . . . . . . 49

4.2 Spreading drop numerical data . . . . . . . . . . . . . . . . 46

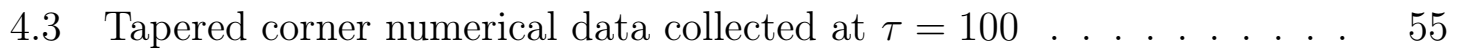

4.4 Tapered corner numerical data . . . . . . . . . . . 56 


\section{List of Symbols}

\begin{tabular}{|c|c|}
\hline$a$ & similarity transform parameter \\
\hline$A$ & cross-sectional flow area \\
\hline$b$ & similarity transform parameter \\
\hline Bo & bond number \\
\hline$c$ & path of line integral \\
\hline$c_{i}$ & power series coefficient \\
\hline$C_{i}$ & constant \\
\hline $\mathcal{C}_{h}$ & constant height $\mathcal{L}$ coefficient \\
\hline $\mathcal{C}_{t}$ & spreading drop $\mathcal{L}$ coefficient \\
\hline $\mathcal{C}_{v}$ & tapered corner $\mathcal{L}$ coefficient \\
\hline$f$ & surface curvature function \\
\hline$F$ & generalized similarity function \\
\hline$F_{a}$ & analytic solution/approximation for $F, F_{a}(\eta)$ \\
\hline$F_{A}$ & cross-flow area function \\
\hline$F_{i}$ & banded flow resistance function \\
\hline$F_{\aleph}$ & numerical approximation for $F$ \\
\hline $\mathcal{F}$ & differential equation, $\mathcal{F}\left(\eta^{+} ; a\right)$ \\
\hline$g$ & acceleration of gravity, $9.81 \mathrm{~m} / \mathrm{s}^{2}$ \\
\hline$g$ & transformed $\boldsymbol{h}$ for finite differencing, $g(Z, \tau)$ \\
\hline$h$ & meniscus height from $z$-axis, $h(z, t)$ \\
\hline$h_{a}$ & analytic solution for $h$ \\
\hline$h_{\aleph}$ & numeric solution for $h$ \\
\hline$H_{l}$ & bulk meniscus height \\
\hline$H_{w}$ & wall height \\
\hline f & transformed meniscus height from $z$-axis, $h(Z, \tau)$ \\
\hline $\mathcal{I}$ & Interpolation function, $\mathcal{I}\left(z_{i}\right)$ \\
\hline$J$ & least squares functional \\
\hline$k$ & time multiplier to balance stability condition \\
\hline $\mathcal{L}$ & tip location, $\mathcal{L}(t)$ \\
\hline$m$ & number of time nodes \\
\hline
\end{tabular}




\begin{tabular}{|c|c|}
\hline$m$ & exponential of $\tau$ analyzed in the tapered corner \\
\hline$n$ & number of space nodes, unless in sigma notation; then $n \in \mathbb{N}$ \\
\hline$O h$ & Ohnesorge number, $\mu /(\sigma \rho H)^{1 / 2}$ \\
\hline$P$ & pressure \\
\hline $\mathcal{Q}$ & residual error \\
\hline$\dot{Q}$ & volumetric flow rate, $\dot{Q}(z, t)$ \\
\hline $\mathcal{R}$ & $\epsilon^{2} \sin ^{4} \alpha / f O h^{2}$ \\
\hline$\Re$ & volume element assumed to be 0 \\
\hline$s$ & infinitesimal length element of line integral \\
\hline $\mathcal{S}$ & sum of $\eta$ terms in perturbed series \\
\hline$t$ & time \\
\hline$t_{f}$ & final time \\
\hline$t_{i}$ & initial time \\
\hline$u$ & $x$-component of velocity \\
\hline $\mathcal{V}$ & flow tip volume \\
\hline$v$ & $y$-component of velocity \\
\hline $\mathscr{V}$ & cross sectional area per unit depth in $z$ \\
\hline$w$ & $z$-component of velocity \\
\hline$x$ & spatial coordinate \\
\hline$y$ & spatial coordinate \\
\hline$z$ & spatial coordinate \\
\hline$Z$ & transformed $z$ \\
\hline$z_{d}$ & distance from $z_{b u l k}$ to tapered corner \\
\hline \multicolumn{2}{|c|}{ Mathematical nomenclature } \\
\hline$D / D t$ & material derivative, $\partial_{t}+u \partial_{x}+v \partial_{y}+w \partial_{z}$ \\
\hline$f$ & general function, which reads "function of" \\
\hline $\mathbb{N}$ & the set of natural numbers $0,1,2 \ldots$ \\
\hline $\mathbb{N}^{+}$ & the set of positive natural numbers $1,2 \ldots$ \\
\hline $\mathcal{O}$ & big O Landau notation \\
\hline $\mathbb{R}$ & the set of real numbers \\
\hline $\mathbb{R}^{+}$ & the set of positive real numbers \\
\hline \multicolumn{2}{|c|}{ Greek Symbols } \\
\hline$\alpha$ & corner half-angle \\
\hline$\Delta \rho$ & change in density \\
\hline
\end{tabular}




$\begin{array}{ll}\Delta \tau & \text { time step } \\ \Delta z & \text { spatial step } \\ \Delta Z & \text { transformed spatial step } \\ \epsilon & \text { fluid column slenderness ration } \\ \varepsilon & \text { small parameter } \\ \eta & \text { generalized similarity parameter } \\ \eta^{+} & \text {invariant generalized similarity parameter } \\ \theta & \text { contact angle } \\ \kappa & \text { dimensionless drop volume } \\ \lambda & \text { invariant transform coefficient } \\ \mu & \text { dynamic viscosity } \\ \pi & \text { ratio of a circle's circumference to radius, } 3.14159 \ldots \\ \rho & \text { fluid density } \\ \sigma & \text { surface tension } \\ \tau & \text { transformed time } \\ \Upsilon & \text { time domain } \\ \phi & \text { trial function, } \phi\left(\eta^{+}\right) \\ \Phi & \text { volume balance constant } \\ \chi & \text { integral best fit measure } \\ \psi & \text { ordinary differential equation to } \mathcal{O}\left(\epsilon^{n}\right) \\ \Omega & \text { domain }\end{array}$

Dimensional Quantities

$H \quad$ characteristic meniscus height

$L \quad$ characteristic length of fluid column

$R \quad$ radius of curvature

$W \quad$ characteristic velocity

Subscripts, Other

$\begin{array}{ll}{\left[\text { sup }^{+}\right.} & \text {invariant transform quantity } \\ {[\text { sup }]^{j}} & \text { time node counter } \\ {[\text { sub }]_{i}} & \text { space node counter } \\ {[\text { sub }]_{t i p}} & \text { quantity evaluated at tip } \\ {[\text { sub }]_{b u l k}} & \text { quantity evaluated at bulk meniscus } \\ {[\text { sub }]_{a}} & \text { analytical or approximated analytical quantity } \\ {[\text { sub }]_{\aleph}} & \text { numerical quantity } \\ \langle\rangle & \text { area-averaged quantity }\end{array}$




\section{Introduction and Motivation}

Capillarity describes a fluid's tendency to flow through (confined) spaces despite the assistance/resistance of external forces. This tendency develops by surface tension wetting and spatial geometry at the molecular level, where an imbalance of adhesive and cohesive forces overpowers other external forces at fluid interfaces. On Earth the capillary magnitude is often measured by the ratio of gravity to surface tension forces, the Bond number $B o \equiv \Delta \rho g H^{2} / \sigma$. Here $\Delta \rho$ is the density change across the fluid interface, $H$ is the characteristic surface length scale, $g$ is the acceleration of gravity $\left(9.81 \mathrm{~m} / \mathrm{s}^{2}\right)$, and $\sigma$ is surface tension. For $B o \gg 1$ gravitational forces dominate flow; for $B o \ll 1$ surface tension dominates flow. If $g$ is sufficiently large, capillary action occurs predominantly at small-scales. In the absence of gravity, such as the environment of space, the Bond number is often small ( $B o \ll 1)$, and hence capillary action can dictate large-scale flows. In fact, Concus and Finn [3] show that capillary action along an infinitely long interior corner can proceed indefinitely when the critical corner wetting condition $\theta<\pi / 2-\alpha$ is satisfied, where $\theta$ is the fluid contact angle and $\alpha$ is half the corner angle.

\subsection{Background Research}

Significant research has been devoted to further understanding capillary-driven flow and its reaction to various geometries. Weislogel [12] investigates such flows subject to irregular polygonal geometries. Weislogel and Lichter [13] demonstrate asymptotic techniques solving capillary flows in interior corners and consider flow throughout a 
container in the limit of long times. Asymptotic solutions present closed form solutions, which are valuable when exploiting optimal flow management designs. Weislogel et al. [11] demonstrate solutions for weakly and passive forced flows of an interior corner and compare critical design criteria. Chen et al. [14] extrapolate sharp corners to rounded interior corners, where analytic and numerical solutions are presented. Convex and concave corner walls are analyzed by Weislogel [9], where negative wall curvature is shown to increase the volumetric flow rate. Asymmetric geometric configurations are documented by Bolleddula et al. [2], where it is found that corner asymmetries can cause chaotic flow migration. Dong and Chatzis [4] analyze flow through a square cross section with both rounded and sharp edges, reporting sharp corners catalyze imbibition and rounded corners buffer imbibition.

\section{$1.2 \quad$ Present Scope}

The aforementioned publications review corner flows in polygonal cylinders. Solely focusing on constant cross-sections excludes a large class of geometries and leaves open questions regarding the bulk meniscus behavior as it approaches the bottom of a container. To better understand fluid behavior and optimize containment we shift focus from cylinders to tapered containers, as illustrated in Figure 1.2. When analyzing fluid behavior in such geometries it is critical to understand the tip location $\mathcal{L}$ as a function of time. We hypothesize that the tapered corner column length is

confined to $t^{2 / 5} \lesssim \mathcal{L} \lesssim t^{1 / 2}$, and in fact that initially the tapered corner regime tip grows as $\mathcal{L} \sim t^{1 / 2}$ and after sufficient time has elapsed its growth transitions to $\mathcal{L} \sim$ $t^{2 / 5}$, as depicted in Figure 1.1. The reason for this hypothesis is explained in Chapter 
4. Validation of this hypothesis begins by deriving the governing tip-displacement equation and its associated boundary conditions, as presented in Chapter 2. Chapter 3 then provides analytical treatment to the capillary rise and spreading drop regimes. These regimes are not arbitrarily chosen, as the tip behavior for the capillary rise $\mathcal{L} \sim t^{1 / 2}$ and spreading drop $\mathcal{L} \sim t^{2 / 5}$ serve as upper and lower bounds respectively for the tapered corner. Since the tapered corner is not well-posed via the employed analytical schemes, a numerical method is presented in Chapter 4. The method is first applied for the capillary rise and spreading drop regimes, where analytical benchmarks are available. The numerical results are shown to converge onto the analytic equations, and we thus employ the method to the tapered corner regime. The results are tabulated, and ultimately we find the tapered corner tip adheres to $t^{2 / 5} \lesssim \mathcal{L} \lesssim t^{1 / 2}$ and the regime in Figure 1.1 is followed for certain geometries, as was expected.

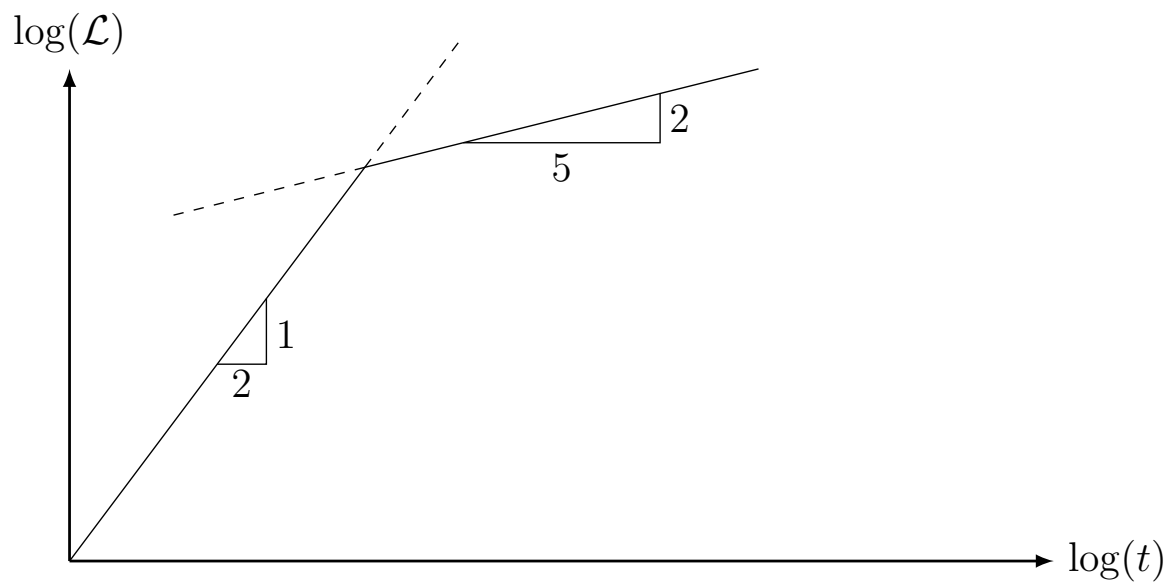

Figure 1.1: A sketch of the hypothesized behavior for the tapered corner $\mathcal{L}$ growth. 


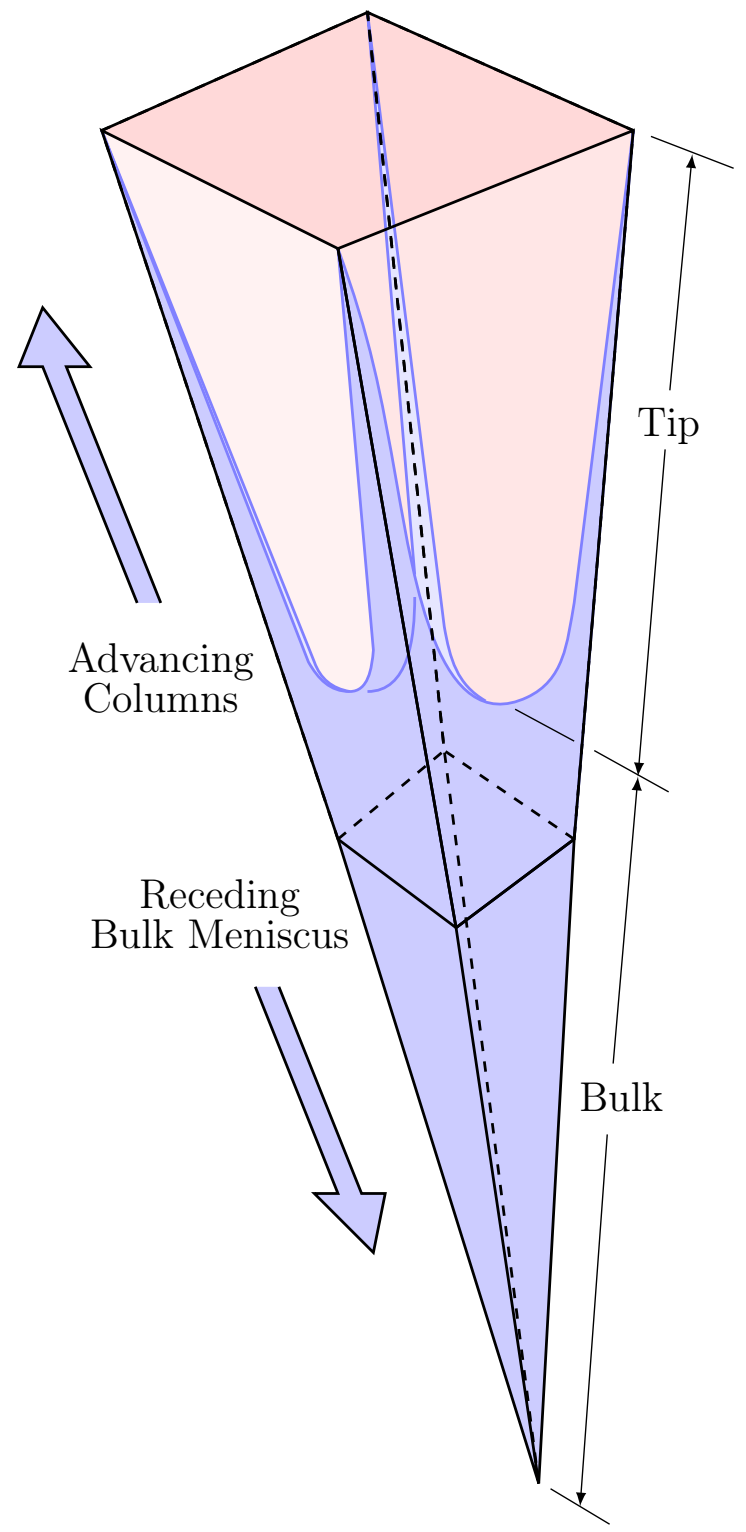

Figure 1.2: Illustration of the tapered corner regime. 


\section{Derivation of Governing Equation}

Since the corner flow dictates most of the flow throughout the container we begin by analyzing this region. Referencing Figure 2.1, $L$ is the characteristic length of the column of fluid along the $z$-axis and $H$ is the characteristic height of the meniscus in the corner along the $x$-axis. The ratio of these length scales $(\epsilon \equiv H / L)$ is crucial in the analysis to follow. Table 2.1 provides other essential, nondimensional quantities.

Table 2.1: Non-dimensional independent and dependent variables and parameters.

\begin{tabular}{|l|l|l|}
\hline Lengths & Velocities & Other \\
\hline$x=x^{\prime} / H$ & $u=u^{\prime} / \epsilon w$ & $P=H f P^{\prime} / \sigma$ \\
$y=y^{\prime} / H \tan \alpha$ & $v=v^{\prime} / \epsilon W \tan \alpha$ & $t=W t^{\prime} / L$ \\
$z=z^{\prime} / L$ & $w=w^{\prime} / W$ & $A=A^{\prime} / H^{2} \tan \alpha$ \\
$\mathcal{L}=\mathcal{L}^{\prime} / L$ & $\langle w\rangle=\langle w\rangle^{\prime} / W$ & $\dot{Q}=\dot{Q}^{\prime} / W H^{2} \tan \alpha$ \\
$h=h^{\prime} / H$ & $W=\epsilon \sigma \sin ^{2} \alpha / \mu f$ & $\epsilon=H / L$ \\
\hline
\end{tabular}

The majority of the dimensionless quantities listed in Table 2.1 are derived through simple geometry. However, a few should be justified. The characteristic $z$-component of velocity $W$ is determined through a balance of pressure and viscous forces. The pressure is scaled via $\sigma / H f: f=f(\alpha, \theta)$, a geometric curvature function of the meniscus in the $x-y$ plane. For $f$ to be a valid scaling measure as outlined by Weislogel [10], the meniscus curvature in the $x^{\prime}-z^{\prime}$ plane must be small, $\epsilon^{2} f \ll 1$. The angle $\alpha$ is half the corner angle and $\theta$ is the contact angle as illustrated in Figure 2.1. It should be noted that the prime ' notation denotes a dimensional quantity throughout the text unless otherwise stated. 


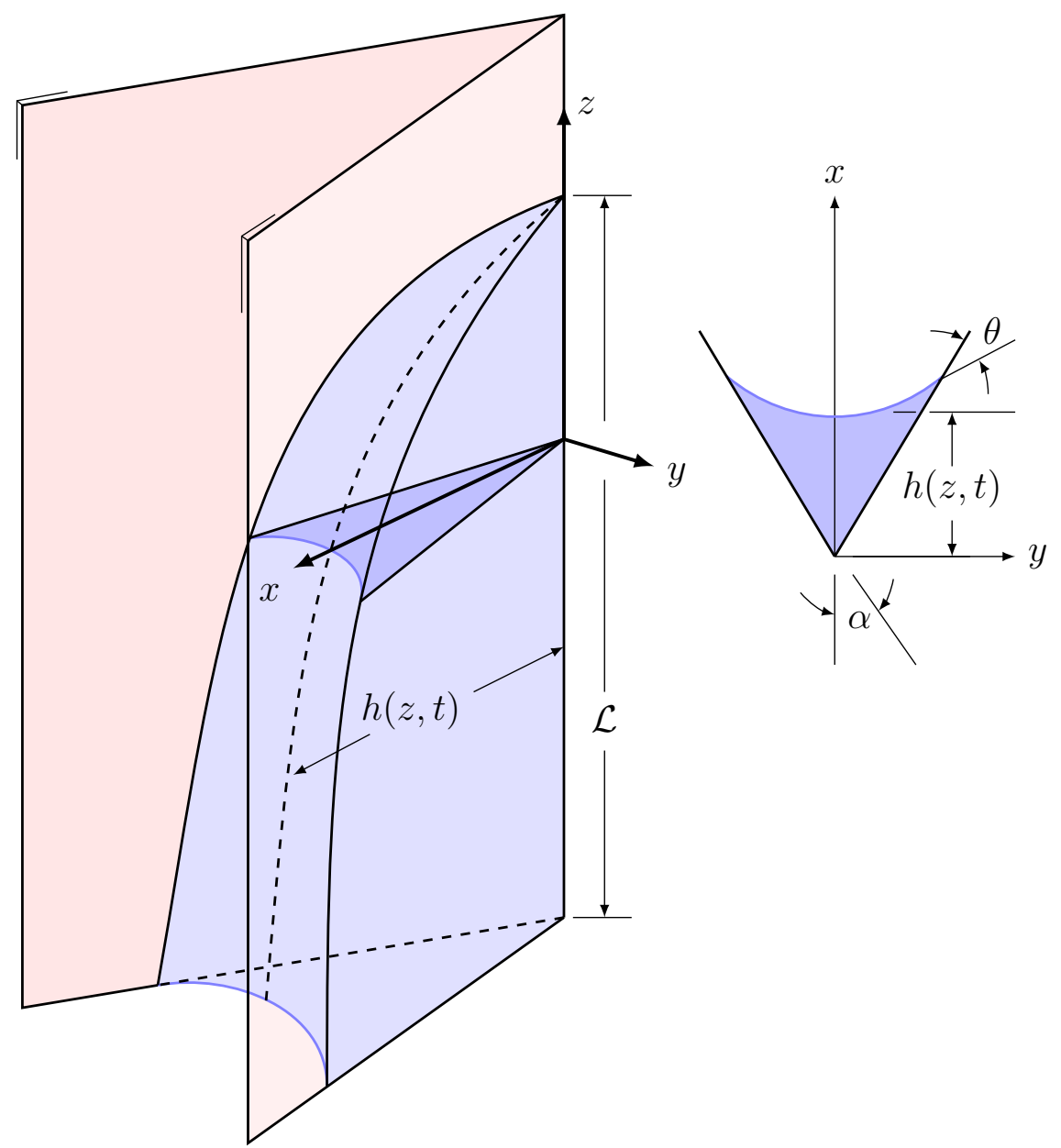

Figure 2.1: Sketch of capillary rise and cross-section in an interior corner.

Ignoring body forces, the dimensionless Navier-Stokes equations governing the tip flow are 


$$
\begin{aligned}
\epsilon^{2} \mathcal{R} \frac{D u}{D t} & =-P_{x}+\epsilon^{2} \nabla^{2} u \\
\epsilon^{2} \mathcal{R}^{2} \tan ^{2} \alpha \frac{D v}{D t} & =-P_{y}+\epsilon^{2} \tan ^{2} \alpha \nabla^{2} v \\
\mathcal{R} \frac{D w}{D t} & =-P_{z}+\nabla^{2} w: \\
\nabla^{2} & \equiv \sin ^{2} \alpha \frac{\partial^{2}}{\partial x^{2}}+\cos ^{2} \alpha \frac{\partial^{2}}{\partial y^{2}}+\epsilon^{2} \sin ^{2} \alpha \frac{\partial^{2}}{\partial z^{2}} \\
\mathcal{R} & \equiv \frac{\epsilon^{2}}{f O h^{2}} \sin ^{4} \alpha .
\end{aligned}
$$

where $D / D t$ is the substantial derivative operator and $O h \equiv \mu /(\sigma \rho H)^{1 / 2}$ is the Ohnesorge number. Boundary conditions for the above equations include a no slip condition along the container wall, zero shear stress along the surface, and a contact angle criterion. These boundary conditions and the Navier-Stokes equations are onerous as posed. However, expanding the dependent variables as $f=f_{0}+\mathcal{O}\left(\epsilon^{2}\right)$ where $f_{0}$ includes $\mathcal{O}(\epsilon)$ terms and assuming $\mathcal{R}=\mathcal{O}\left(\epsilon^{2}\right)$ reduces (2.3) to first order as

$$
\frac{\partial P_{0}}{\partial z}=\sin ^{2} \alpha \frac{\partial^{2} w_{0}}{\partial x^{2}}+\cos ^{2} \alpha \frac{\partial^{2} w_{0}}{\partial y^{2}}
$$

Solving (2.6) yields an expression for $\left\langle w_{0}\right\rangle$ as

$$
\left\langle w_{0}\right\rangle=-F_{i} h^{2} \frac{\partial P_{0}}{\partial z}=-F_{i} \frac{\partial h}{\partial z}
$$

where $F_{i}$ is a geometric parameter governing the curvature of the meniscus. A mass 
balance in the $z$ direction yields

$$
\frac{\partial A}{\partial t}=-\frac{\partial \dot{Q}}{\partial z}=-\frac{\partial}{\partial z} A\langle w\rangle .
$$

Introducing the cross-sectional area function $F_{A} \equiv A^{\prime} / h^{\prime 2}$, where $F_{A}=f(\alpha, \theta)$, and substituting $F_{A}$ and (2.7) into (2.8) yields the leading order governing corner flow equation

$$
2 \frac{\partial h}{\partial t}=F_{i}\left(2\left(\frac{\partial h}{\partial z}\right)^{2}+h \frac{\partial^{2} h}{\partial z^{2}}\right) .
$$

Defining $\tau \equiv F_{i} t / 2$ reduces (2.9) to

$$
\frac{\partial h}{\partial \tau}=h \frac{\partial^{2} h}{\partial z^{2}}+2\left(\frac{\partial h}{\partial z}\right)^{2} .
$$

The first associated boundary condition is zero height at the tip location $z=\mathcal{L}$,

$$
h(\mathcal{L}(\tau), \tau)=0 .
$$

The second boundary condition is prescribed by flow conditions. In almost all cases the second boundary condition requires information about the bulk meniscus location, introducing a closure issue. A conservation of mass integral closes (2.10) when the bulk meniscus location appears as a boundary condition, as outlined in Appendix B and to be shown in Section 4.1.3. Once defining the second boundary condition, all that remains is determining an initial condition. It should be noted 
while volume is conserved over the tip and bulk regions, (2.10) only governs the displacement for the tip region, as depicted in Figure 1.2. Then (2.10) does not imply volume is conserved for an arbitrary control volume. Equation (2.10) governs tip displacement and bulk meniscus location, and is the fundamental equation hereafter analyzed. Note (2.10) is only valid along the advancing column and does not hold for the bulk meniscus. Three different flow regimes are studied herein, where each arises from different boundary conditions ${ }^{1}$.

\footnotetext{
${ }^{1}$ To this point only one boundary condition, (2.11), for (2.10) is presented. However, (2.10) requires two. The second boundary condition is a property of a particular flow problem. Since three problems are studied, three boundary conditions are considered.
} 


\section{Analytics}

This chapter is devoted to analytically solving (or at least approximating) solutions to equation (2.10). In its current form, (2.10) is difficult to solve and many solution methods fail. Weislogel [10] and Ramé and Weislogel [6] apply similarity transforms to (2.10), transforming it into a tractable ODE, where at least one exact solution exists for the appropriate boundary and initial conditions. The beginning of this chapter summarizes and applies that transform. Since both the capillary rise and spreading drop regimes are well-posed in the sense that non-conflicting boundary conditions are established after transformation, it is possible to attain analytical expressions for each. As will be shown, an exact analytic solution for the capillary rise regime is unknown, and must then be approximated. Two approximation techniques are presented: perturbations in the exponent of a power-law similarity transform and a least squares variational calculus method. The variational calculus method was introduced by Becker [1]; the power law perturbational technique is original work.

\subsection{Similarity Transform}

Due to the nonlinearity of equation (2.10) typical solution methods (separation of variables, etc.) fail. Rather than separating $h$ into products of functions of $\tau$ and $z$, a similarity transform consolidates variables, reducing the PDE to an ODE. We introduce

$$
h=C_{1} \tau^{a} F(\eta) \quad \eta=C_{2} z \tau^{b} \quad \mathcal{L}=\eta_{t i p} C_{2}^{-1} \tau^{-b}
$$


where $C_{1}, C_{2}$ and $\eta_{t i p}$ are constants. Equation (3.1) recasts (2.10) as

$$
F F_{\eta \eta}+2 F_{\eta}^{2}-\frac{\tau^{-1-a-2 b}}{C_{1} C_{2}^{2}}\left[a F+b \eta F_{\eta}\right]=0 .
$$

Similarity is achieved when (3.2) is $\tau$ independent, which implies $-1-a-2 b=0$. Before proceeding, it is helpful to notice (3.2) is invariant under the following transformations

$$
F=\lambda^{2} F^{+} \quad \eta=\lambda \eta^{+} .
$$

Applying the similarity transform (3.1) to $(2.11)$ yields $F\left(\eta_{t i p}\right)=0$, where (3.3) implies $F^{+}\left(\lambda \eta_{t i p}^{+}\right)=0$. Since $\lambda$ is arbitrary let $\lambda=\eta_{t i p}$, which implies $\eta_{t i p}^{+}=1$. Then one boundary condition for (3.2) (once transformed from $F \mapsto F^{+}$) is

$$
F^{+}(1)=0 .
$$

Letting $\eta=\eta_{t i p}$ in (3.2) and then applying (3.3) yields a second boundary condition

$$
F_{\eta^{+}}^{+}(1)=\frac{b}{2 C_{1} C_{2}^{2}} .
$$

Two boundary conditions at the tip have been derived, and a backward $(\eta \rightarrow-\infty)$ shooting Runge Kutta method can now solve the system if the constants $a, b, C_{1}$, and $C_{2}$ are known. Determining these constants requires physical assumptions, which are 
discussed below.

\subsubsection{Capillary Rise (Constant Height)}

The capillary rise flow regime develops from a volume of fluid in static equilibrium. During, say, a typical drop tower test, gravity (which operates in the $-z$ direction) is suddenly absent, causing a force imbalance. The under pressure in the liquid caused by local wetting (Concus and Finn [3]) causes the fluid to wick in the $z$ direction into and along the corner. Experiments show this regime quickly establishes a constant height at some defined $z=0$, and while not obvious, $h(0, \tau)=1[10]$. Notice $h(0, \tau)=1$ implies $F(0)=C_{1}^{-1} \tau^{-a}$, and thus $a=0$ to maintain self-similarity. Then $b=-1 / 2$ and we choose constants $C_{1}=1$ and $C_{2}=2^{-1 / 2}$, which implies $F(0)=1$. Equation $(3.1)$ becomes $h=F, \eta=z(2 \tau)^{-1 / 2}$, and $\mathcal{L}=\eta_{\text {tip }}(2 \tau)^{1 / 2}$. Then (3.2) becomes (after transforming $F \mapsto F^{+}$)

$$
F^{+} F_{\eta^{+} \eta^{+}}^{+}+2{F_{\eta^{+}}^{+}}^{2}+\eta^{+} F_{\eta^{+}}^{+}=0 .
$$

subject to

$$
F^{+}(1)=0 \quad F_{\eta^{+}}^{+}(1)=-\frac{1}{2} .
$$

Equation (3.6) is now well-posed and can be numerically solved for $F^{+}\left(\eta^{+}\right)$. The invariant parameter $\lambda$ may be solved for by transforming $F(0)=1 \mapsto F^{+}(0)=\lambda^{-2}$. 
Then $\mathcal{L}$ can be expressed as

$$
\mathcal{L}=\mathcal{C}_{\hbar} \tau^{1 / 2}: \mathcal{C}_{\hbar} \equiv 2.407 \ldots
$$

With $\lambda$ known it is possible to transform $F^{+}\left(\eta^{+}\right) \mapsto F(\eta)$ via (3.3). Table 3.1 tabulates significant values for $F(\eta)$ where calculations have been computed in Mathematica 10.1.

Table 3.1: Summarized values from the similarity solutions. Results were initially calculated by Weislogel [10] and are here verified in Mathematica 10.1.

\begin{tabular}{|c|c|c|c|c|}
\hline Case & $\eta_{t i p}=\lambda$ & $F(0)$ & $F_{\eta}(0)$ & $F_{\eta}\left(\eta_{t i p}\right)$ \\
\hline Capillary Rise $(a=0)$ & $1.702 \ldots$ & 1 & $-0.349 \ldots$ & $-0.851 \ldots$ \\
\hline Spreading Drop $(a=-1 / 5)$ & $1.496 \ldots$ & $1.119 \ldots$ & 0 & $-1.496 \ldots$ \\
\hline
\end{tabular}

\subsubsection{Spreading Drop (Constant Volume)}

The spreading drop regime develops from a drop of fluid of fixed volume $H^{3}$ instantly appearing on an interior corner at some location defined as $z=0$. The drop is symmetric in the $x-z$ plane about the $x$ axis and disperses evenly. A conservation of mass integral ${ }^{2}$ gives $a=-1 / 5, b=-2 / 5, C_{1}=\left(\kappa^{2} / 5\right)^{1 / 5}, C_{2}=\left(5^{2} \kappa\right)^{-1 / 5}$ where $\kappa \equiv \epsilon / 2 F_{A}$. Then (3.2) reduces to (after transforming $F \mapsto F^{+}$)

$$
F^{+} F_{\eta^{+} \eta^{+}}^{+}+2{F_{\eta^{+}}^{+2}}^{2}+\eta^{+} F_{\eta^{+}}^{+}+\frac{1}{2} F^{+}=0
$$

\footnotetext{
${ }^{2}$ See Appendix A for details.
} 
subject to

$$
F^{+}(1)=0 \quad F_{\eta^{+}}^{+}(1)=-1 .
$$

Before turning to a numerical solution, the following ansatz is advanced.

Ansatz 1. Equation (3.9) may be solved exactly via a quadratic in $\eta^{+}$. When subjecting said quadratic to (3.10) the exact solution is

$$
F^{+}\left(\eta^{+}\right)=\frac{1}{2}\left(1-\eta^{+2}\right)
$$

Notice (3.1) can be expressed as $h=\kappa^{2 / 5}(5 \tau)^{-1 / 5} F, \eta=\kappa^{-1 / 5} z(5 \tau)^{-2 / 5}$. It can be shown $^{3} \lambda=\left(\int_{0}^{1} F^{+}\left(\eta^{+}\right)^{2} d \eta^{+}\right)^{-1 / 5}=(15 / 2)^{1 / 5} \doteqdot 1.496 \ldots$ Now $\mathcal{L}$ may be expressed as

$$
\mathcal{L}=\mathcal{C}_{v} \tau^{2 / 5}: \mathcal{C}_{v} \equiv \frac{3^{1 / 5} 5^{3 / 5}}{2^{2 / 5}} \frac{\epsilon}{F_{A}} \doteqdot 0.9872 \ldots
$$

where we let $\epsilon / F_{A}=1 / 100$. With $\lambda$ known, (3.3) can transform $F^{+}$back to $F$. Using (3.1) to back-transform $F \mapsto h$ yields the following exact integral solution for $(2.10)$ subject to both (2.11) and a constant volume boundary condition ${ }^{4}$ :

$$
h=\frac{1}{2} \lambda^{2} \kappa^{2 / 5}(5 \tau)^{-1 / 5}\left(1-\frac{\eta^{2}}{\lambda^{2}}\right)
$$

\footnotetext{
${ }^{3}$ See Appendix A for details.

${ }^{4}$ The constant volume boundary condition mentioned is $h_{z}(0, \tau)=0$, which is explained in detail in Section 4.1.2.
} 
where $0 \leq \eta \leq \lambda$. Table 3.1 tabulates significant values for $F(\eta)$, where calculations have been computed in Mathematica 10.1.

\subsubsection{Universal Similarity}

Before continuing, it will be useful to rewrite (3.2) as a self-similar equation generalized for all $a$ values. Thus let $b=-(1+a) / 2, C_{1}=1$ and $C_{2}=|b|^{1 / 2}$. Then (3.2) is rewritten as

$$
\mathcal{F}\left(\eta^{+} ; a\right) \equiv F^{+} F_{\eta^{+} \eta^{+}}^{+}+2{F_{\eta^{+}}^{+2}}^{2}+\eta^{+} F_{\eta^{+}}^{+}-\frac{2 a}{1+a} F^{+}=0 .
$$

Applying the invariant transformation (3.3) to the boundary conditions, (3.5) and (3.4) are respectively written as

$$
F_{\eta^{+}}^{+}(1)=-1 / 2 \quad F^{+}(1)=0 .
$$

Then it is clear the only undetermined similarity variable is $a$. In fact, $a$ dictates the physical flow regime that equation (3.2) describes and also impacts existence of solutions. Notice the similarity constants defined in this section, while consistent for Section 3.1.1, differ from those suggested in Section 3.1.2. $\mathcal{F}\left(\eta^{+}, 0\right)$ yields a similar exact solution as (3.11), which is

$$
F^{+}\left(\eta^{+}\right)=\frac{1}{4}\left(1-\eta^{+2}\right) .
$$

Distinguishing (3.16) and (3.11) is necessary for employing the perturbational 
technique, as both the spreading drop and the capillary rise regimes must be cast identically.

\subsection{Analytical Solutions and Approximations}

In Section 3.1 an exact solution for the spreading drop regime was recovered. However, no analytical solution was found for the capillary rise regime. Without an analytical expression, it is not possible to back-transform $F \mapsto h$ for all $\eta \in[0, \mathcal{L}]$. In this section we attempt to analytically approximate solutions to (3.14) via perturbing the power law exponent $a$. The perturbational approximation is similar to a Taylor series: as a Taylor series sums polynomial terms to approximate functions, this technique sums truncated analytic solutions to approximate non-analytic solutions. Similar to a Taylor series' validity on a radius of convergence, the perturbational technique is valid when approximated from a sufficiently close neighboring analytic solution. Succinctly stated, this technique perturbs exact solutions so as to analytically approximate nearby numerical solutions wherein no analytic solution exists.

\subsubsection{The Perturbational Technique}

Equation (3.13) confirms the existence of at least one exact analytic solution for (2.10). In fact, exact solutions exist not only for the spreading drop $a=-1 / 5$ regime, but also for other flow regimes where $a=-1 / 3$ and $a=1$ [10]. However, no exact solution has been found for the capillary rise $a=0$ regime. Despite not having an exact solution, the capillary rise regime, along with numerous other $a$ values, can 
be analytically approximated by perturbing a well-understood ${ }^{5}$ similarity transform exponent $a$ in (3.1) by some $\varepsilon \in \mathbb{R}^{+}: \varepsilon \ll 1$. Denote this well-understood $a$ value $a_{*}$, and denote the solution's $a$ value we seek to analytically approximate $a_{\dagger}$. The perturbation is then $a_{\dagger}=a_{*}+\varepsilon$, which implies the similarity transform (3.1) is redefined as $h=C_{1} \tau^{a} F(\eta) \mapsto h=C_{1} \tau^{a+\varepsilon} F(\eta)$. The method follows.

Define $a_{*} \in \mathbb{R}$ as an $a$ value such that $\mathcal{F}^{+}\left(\eta^{+}, a_{*}\right)$ implies $F_{*}^{+}\left(\eta^{+}\right)$exists and is analytic. Define $a_{\dagger} \in \mathbb{R}$ as an $a$ value such that $\mathcal{F}^{+}\left(\eta^{+}, a_{\dagger}\right)$ implies $F_{\dagger}^{+}\left(\eta^{+}\right)$exists (although not necessarily known nor analytic). To approximate $F_{\dagger}^{+}\left(\eta^{+}\right)$via $F_{*}^{+}\left(\eta^{+}\right)$, first select an $a_{*}$ such that $\left|a_{\dagger}-a_{*}\right|$ is minimized. Define $F_{0}^{+}$as $F_{*}^{+}\left(\eta^{+}\right)$solving the differential equation corresponding to $a_{*}$. Now make a naive expansion in (3.14), letting

$$
F^{+}\left(\eta^{+}\right)=\sum_{\mathbb{N}} \varepsilon^{n} F_{n}^{+}\left(\eta^{+}\right) .
$$

Perturbing $a_{*}=a_{\dagger}+\varepsilon$ implies from equation (3.14) the term $-2 a /(1+a)=f(\varepsilon)$. Since (3.14) will be weighted in $\varepsilon$ make a McLaurin expansion in $\varepsilon$ for $-2\left(a_{*}+\varepsilon\right) /(1+$ $\left.a_{*}+\varepsilon\right)=-2 a_{*} \sum_{\mathbb{N}} \varepsilon^{n} /\left(1+a_{*}\right)^{n+1}$. Substituting the naive and McLaurin expansions

\footnotetext{
${ }^{5}$ Well understood implies all $a$ values whose corresponding solutions that exactly solve (3.14), which is currently values $-1 / 3,-1 / 5$, and 1 , the similarity flow regimes with exact solutions.
} 
into (3.14) yields

$$
\begin{aligned}
& \sum_{\mathbb{N}} \varepsilon^{n} F_{n}^{+\prime \prime} \sum_{\mathbb{N}} \varepsilon^{n} F_{n}^{+}+2\left(\sum_{\mathbb{N}} \varepsilon^{n} F_{n}^{+\prime}\right)^{2}+\eta^{+} \sum_{\mathbb{N}} \varepsilon^{n} F_{n}^{+\prime}+ \\
& -2 a_{*} \sum_{\mathbb{N}} \varepsilon^{n}\left(1+a_{*}\right)^{-(n+1)} \sum_{\mathbb{N}} \varepsilon^{n} F_{n}^{+}=0
\end{aligned}
$$

where prime ' notation here denotes an ordinary derivative with respect to $\eta^{+}$. Weighting (3.18) in order $\varepsilon$ gives rise to a system of ordinary differential equations, where each successive equation to $\mathcal{O}\left(\varepsilon^{n}\right)$ is denoted $\psi_{n}$ and is predicated on the solution to the previous system $\left[\psi_{0}, \ldots, \psi_{n-1}\right]$. By construction it will always be the case that $\psi_{0}$ is solved by $F_{0}^{+}$, and in general $\psi_{n}$ is solved by the inclusive set of $n+1$ solutions $\left\{F_{0}^{+}, \ldots, F_{n}^{+}\right\}$. Then it is clear if $F_{0}^{+}$is known, $F_{1}^{+}$may be solved and inductively $F_{n}^{+}$ may also be solved. With $F_{n}^{+}$known, $F^{+}\left(\eta^{+}\right)$is known from (3.17) through accuracy of $\mathcal{O}\left(\varepsilon^{n}\right)$. It remains to determine $\varepsilon$, which we define as $\varepsilon \equiv a_{\dagger}-a_{*}$. It is interesting that given $F_{n}^{+}, \psi_{n+1}$ is linear despite the nonlinear nature of (3.18).

The component $\psi_{n}$ is a second order ODE, and thus requires 2 boundary conditions. The above analysis suggests boundary conditions for $F_{0}^{+}$are valid for $\psi_{0}$. Solving successive equations requires more boundary conditions, and in general the system $\left[\psi_{0}, \ldots, \psi_{n}\right]$ requires $2(n+1)$ boundary conditions. Each set of boundary conditions is found by applying the naive expansion $F^{+}\left(\eta^{+}\right)=\sum_{\mathbb{N}} F_{n}^{+}\left(\eta^{+}\right) \varepsilon^{n}$ to (3.15) and weighting to $\mathcal{O}\left(\varepsilon^{n}\right)$.

Example 1. Using the method described above, approximate the solution for any a 
in an $\varepsilon$ neighborhood of $a=-1 / 5$ through $\mathcal{O}(\varepsilon)$ accuracy.

Proof. Substitute $a_{*}=-1 / 5$ into (3.18) and expand to $\mathcal{O}\left(\varepsilon^{0}\right)$, which yields

$$
\psi_{0} \equiv F_{0}^{+\prime \prime} F_{0}^{+}+2\left(F_{0}^{+^{\prime}}\right)^{2}+\eta^{+}{F_{0}^{+\prime}}^{+} \frac{1}{2} F_{0}^{+}=0
$$

Recall (3.16) solves (3.19); thus $\psi_{0}$ is solved. To solve for $F_{1}^{+}$, rewrite (3.18) at $\mathcal{O}(\varepsilon)$

$$
\psi_{1} \equiv-\frac{25 F_{0}^{+}}{8}+\frac{F_{1}^{+}}{2}+\left(\eta^{+}+4{F_{0}^{+}}^{\prime}\right){F_{1}^{+\prime}}^{+} F_{1}^{+}{F_{0}^{+\prime \prime}}^{+} F_{0}^{+} F_{1}^{+\prime \prime}=0
$$

which is linear in $F_{1}^{+}$. Associated boundary conditions at $\mathcal{O}(\varepsilon)$ are then

$$
\begin{gathered}
\sum_{\mathbb{N}} F_{n}^{+}(1) \varepsilon^{n}=0 \Longrightarrow F_{1}^{+}(1)=0 \\
\sum_{\mathbb{N}} F_{n}^{+^{\prime}}(1) \varepsilon^{n}=-\frac{1}{2} \Longrightarrow F_{1}^{+^{\prime}}(1)=0 .
\end{gathered}
$$

Solving (3.20) via Mathematica 10.1 analytically yields

$$
\begin{aligned}
& F_{1}^{+}\left(\eta^{+}\right)= \\
& \frac{5\left(1+3 \eta^{+2}+3 \eta^{+3}+\eta^{+}(\log 256-7)+\log 256-8\left(1+\eta^{+}\right) \log \left(1+\eta^{+}\right)\right)}{48\left(1+\eta^{+}\right)} .
\end{aligned}
$$

To better understand (3.23) and easily operate over it, make a Taylor series expansion centered around $\eta^{+}=1$, which solves the boundary conditions identically. 
Then (3.23) is expressed as

$$
F_{1}^{+}\left(\eta^{+}\right)=\frac{25}{48}\left(\eta^{+}-1\right)^{2}-\frac{25}{288}\left(\eta^{+}-1\right)^{3}+\mathcal{O}\left(\left(\eta^{+}-1\right)^{4}\right)
$$

where the Lagrange Remainder Theorem ${ }^{6}$ may be used to approximate the error of (3.24). $F^{+}\left(\eta^{+}\right)$is then approximated as

$$
F^{+}\left(\eta^{+}\right) \approx \frac{1}{4}\left(1-\eta^{+^{2}}\right)+\varepsilon\left[\frac{25}{48}\left(\eta^{+}-1\right)^{2}-\frac{25}{288}\left(\eta^{+}-1\right)^{3}\right]+\mathcal{O}\left(\varepsilon^{2}\right)
$$

where $\varepsilon=a_{\dagger}+1 / 5$. To approximate an analytical solution simply choose an $a_{\dagger}$.

The spreading drop solution can be perturbed to approximate the capillary rise similarity solution, thus $a_{\dagger}=0$. Figure 3.1 plots this perturbation through $\mathcal{O}\left(\varepsilon^{7}\right)$. Table 3.2 tabulates relevant numerical data from the perturbational technique already described. As $n$ increases, the maximum error diminishes with successive sums in a decaying exponential manner, as Figure 3.2 illustrates. Evidently perturbing the similarity transform by some constant $\varepsilon=1 / 5$ does remarkably well at approximating the numerical solution to (3.6), which was solved in Mathematica 10.1.

\footnotetext{
${ }^{6}$ Consult Spivak [8] for details.
} 


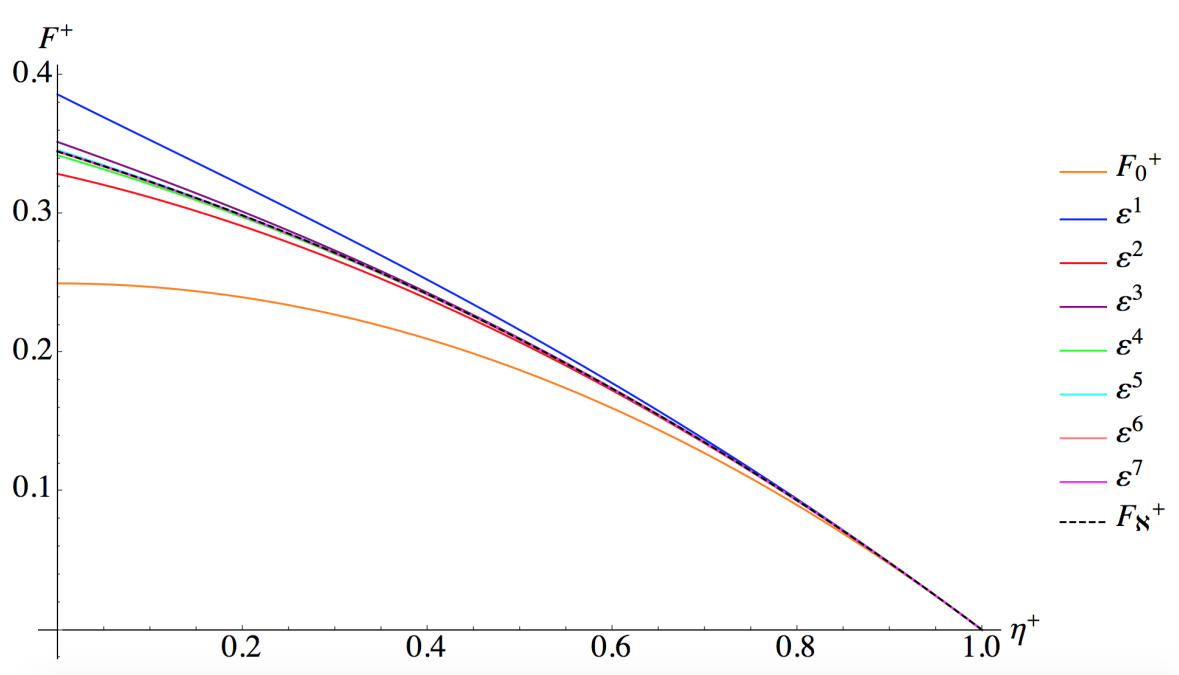

Figure 3.1: The naive expansion (3.17) is plotted through various orders of $\varepsilon$ against a numerical solution denoted $F_{\aleph}^{+}$solved in Mathematica 10.1. $\varepsilon^{n}$ denotes $\sum F_{n} \varepsilon^{n}$. $F_{0}^{+}$is (3.16), the exact solution for the spreading drop regime subject to generalized similarity constants, as outlined in Section 3.1.3. 


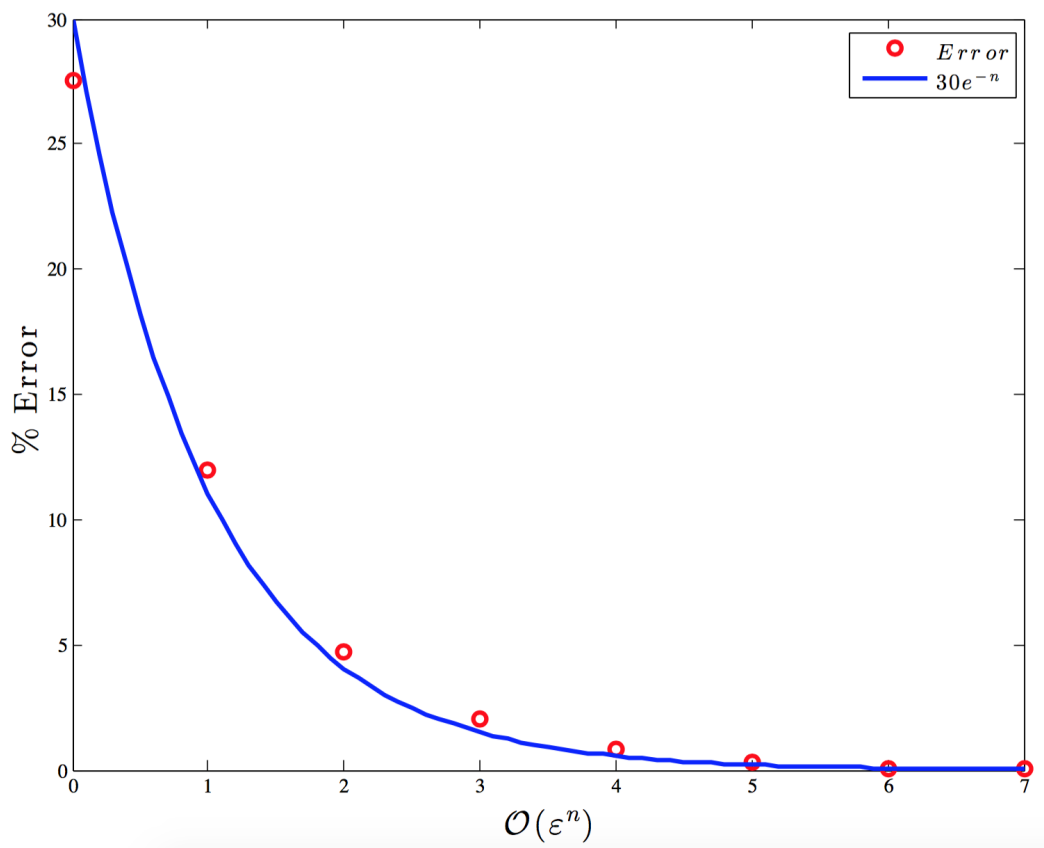

Figure 3.2: Maximum error for $\eta^{+} \in[0,1]$ for $F_{a}=\sum F_{n} \epsilon^{n}$ plotted against $n$. Error is calculated as $100\left[F_{a}(0)-F_{\aleph}(0)\right] / F_{a}(0)$, where $F_{\aleph}$ is the corresponding numerical solution for the capillary rise regime, which is calculated in Mathematica 10.1. An exponential plot is overlaid. 
Table 3.2: Capillary rise analytically approximated data, where $F_{a}=\sum F_{n} \epsilon^{n}$ and $F_{\aleph}$ is a numerical solution to for the capillary rise regime via Mathematica 10.1. $\chi$ is defined in Appendix D as a measure of fit. Max \% Error is defined as $100\left[F_{a}(0)-F_{\aleph}(0)\right] / F_{a}(0): \eta^{+} \in[0,1]$.

\begin{tabular}{|c|c|c|}
\hline$\sum_{n} F_{n} \epsilon^{n}$ & $\chi$ & Max \% Error \\
\hline$n=0$ & 0.0305931 & -27.5705 \\
\hline$n=1$ & 0.0110736 & 11.9348 \\
\hline$n=2$ & 0.00394172 & -4.68234 \\
\hline$n=3$ & 0.00139808 & 1.99493 \\
\hline$n=4$ & 0.00064304 & -0.787077 \\
\hline$n=5$ & 0.000119009 & 0.323315 \\
\hline$n=6$ & 0.000146429 & -0.0794701 \\
\hline$n=7$ & 0.0000568437 & 0.0605253 \\
\hline$n \rightarrow \infty$ & $\rightarrow 0$ & $\rightarrow 0$ \\
\hline
\end{tabular}

The perturbational technique has far reaching, unusual implications with respect to the family of differential equations (2.10) describes. We demonstrated the method's utility in approximating a similarity solution for $a=0$. The parameter $a=0$ was derived from the physical capillary rise regime, which was well-posed in the similarity transform. However, other physical regimes may not be well-posed and hence leave corresponding $a$ values undetermined, if they exist at all. If such a regime (i.e. the tapered corner) is believed to be constrained by a regime whose known $a$ value exists (capillary rise and spreading drop), then it is possible to approximate such a regime's analytical solution by perturbing the known $a$ value and checking the results numerically. Should this method work, other regimes that are known to be bounded 
by the newly approximated $a$ value could also be found, and inductively many flow regimes could potentially be analytically described.

\subsection{Variational Methods}

The technique discussed in Section 3.2.1 approximates solutions to (3.14) but requires a nearby exact solution. Exact solutions are rarely available; then it is instructive to ascertain an approximate analytic solution without knowing any exact solutions. When seeking a non-exact solution, an ideal requirement is to minimize the error of the sought after solution with respect to the exact solution. It is then natural to consider methods from the calculus of variations, specifically a least squares variational technique. ${ }^{7}$ The method follows.

Choose a trial quadratic function to solve (3.14) defined as

$$
\phi\left(\eta^{+}\right) \equiv A \eta^{+^{2}}+B \eta^{+}+C .
$$

Equation (3.26) is not chosen arbitrarily, as a quadratic analytically solves the spreading drop regime. Equation (3.26) must satisfy (3.15), thus $B$ and $C$ are known unique functions of $A$. Then (3.26) can be expressed as

$$
\phi\left(\eta^{+}\right) \equiv \frac{1}{2}+A-\left(\frac{1}{2}+2 A\right) \eta^{+}+A{\eta^{+}}^{2}: A=f(a) .
$$

\footnotetext{
${ }^{7}$ For a theoretical description consult Becker [1]. For employment of said theory, consult Mayer [5], where the non-linear diffusion equation is approximated.
} 
A measurement of validity for (3.27) when approximating (3.14) is given by the residual

$$
\mathcal{Q} \equiv \phi \phi^{\prime \prime}+2 \phi^{2}+\eta^{+} \phi^{\prime}-\frac{2 a}{1+a} \phi
$$

where prime' notation denotes an ordinary derivative. Notice $\mathcal{Q}=f\left(\eta^{+} ; a, A\right)$. However, solving for a particular flow regime implies $a$ is known. Then the resulting functional is

$$
J(A)=\int_{0}^{1} \mathcal{Q}^{2} \mathrm{~d} \eta^{+}
$$

where we wish to minimize $J=f(A)$; thus take $J^{\prime}(A)=0$. An optimal $A$ is now known, and an approximate solution $\phi\left(\eta^{+}\right)$to (3.14), given a flow parameter $a$, is found.

Applying the aforementioned variational technique to approximate $\mathcal{F}\left(\eta^{+}, 0\right)$ yields

$$
\phi\left(\eta^{+}\right)=0.352223-0.204446 \eta^{+}-0.147777 \eta^{+2} .
$$

Figure 3.3 plots both (3.30) and a numerical solution to $\mathcal{F}\left(\eta^{+}, 0\right)$. Note $\phi\left(\eta^{+}\right)$from (3.30) equals (3.16) for $a=-1 / 5$. 


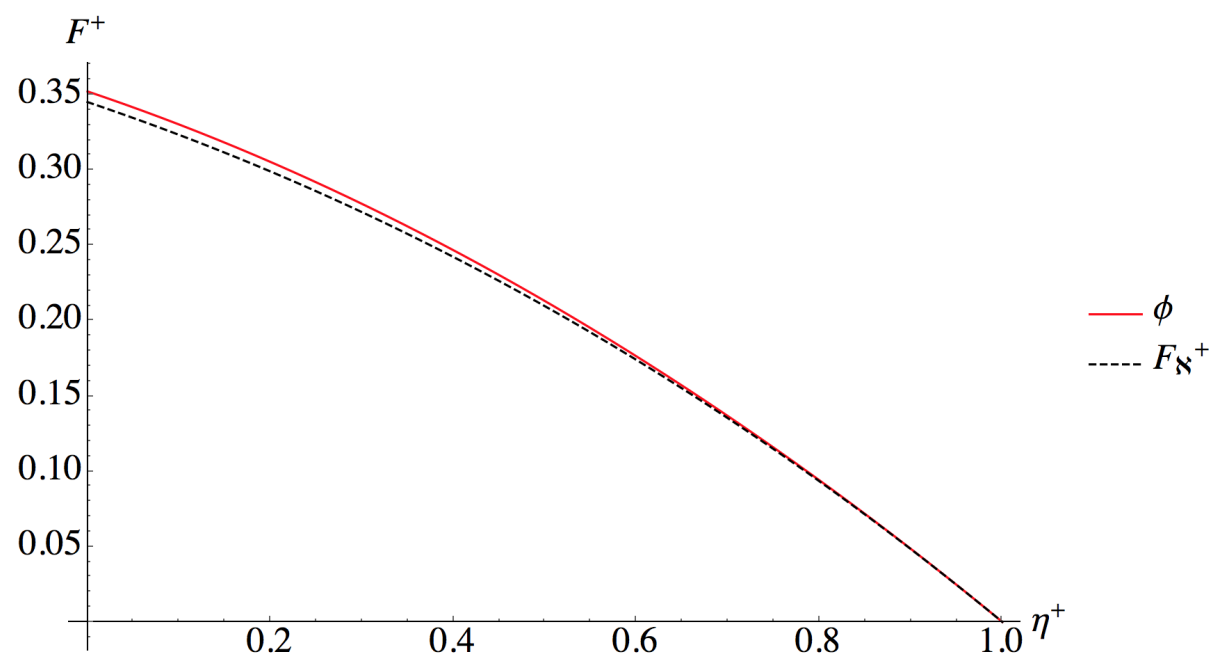

Figure 3.3: $\phi\left(\eta^{+}\right)$is plotted plotted against a numerical solution denoted $F_{\aleph}^{+}$solved in Mathematica 10.1. The maximum error is $2.0 \%$ for $\eta^{+}=0$ and is defined as $100\left[\phi(0)-F_{\aleph}(0)\right] / \phi(0)$. 


\subsection{Summary}

To summarize, Chapter 2 concluded with the development of a second order, nonlinear partial differential equation with one dynamic boundary condition. Chapter 3 began by transforming this PDE into an ODE via a similarity transform. Two regimes were considered: the spreading drop and the capillary rise regime. An exact solution was found for the spreading drop regime. Since no exact solution was found for the capillary rise regime, the second half of Chapter 3 developed a perturbational technique to analytically approximate an exact solution. A well-understood variational calculus technique was then employed to approximate an analytical solution

for the capillary rise regime. These approximations were developed for benchmarking numerical work to be introduced next. 


\section{Numerical Analysis and Results}

Due to the non-linearity and dynamic boundary condition of (2.10), an analytical solution has not generally been found ${ }^{8}$. In fact, as was shown in the constant height regime, even if boundary conditions are well defined, analytical solutions remain unknown. For this reason attention is given to numerical methods for solutions. This chapter begins by discretizing (2.10) via a forward time, centered space, finite difference scheme. Three cases will be analyzed: the spreading drop, capillary rise, and tapered corner. Numerical results for the former two schemes are benchmarked with analytical solutions/approximations as found in Chapter 3. Since the tapered corner is not well-posed in the similarity transform, no benchmark is available.

\subsection{Generalized Finite Difference Scheme}

Notice (2.10) can be rewritten as

$$
h \frac{\partial h}{\partial \tau}=h^{2} \frac{\partial^{2} h}{\partial z^{2}}+2 h\left(\frac{\partial h}{\partial z}\right)^{2}=\frac{\partial}{\partial z}\left(h^{2} \frac{\partial h}{\partial z}\right) \Longrightarrow \frac{\partial\left(h^{2}\right)}{\partial \tau}=\frac{2}{3} \frac{\partial^{2}\left(h^{3}\right)}{\partial z^{2}} .
$$

To normalize (2.11), apply the transformation

$$
Z=\frac{z}{\mathcal{L}(\tau)}
$$

\footnotetext{
${ }^{8}$ Having only one boundary condition implies $(2.10)$ is underspecified. The term "generally" is used to account for all possible boundary conditions.
} 
which yields a transformed boundary condition

$$
h(1, \tau)=0
$$

Note $h(z, \tau) \mapsto \mathfrak{h}(Z, \tau)$. The total differential of $\boldsymbol{h}$ is then expressed as

$$
\mathrm{d} \hat{h}=\left(\frac{\partial \hat{h}}{\partial \tau}\right)_{Z} \mathrm{~d} \tau+\left(\frac{\partial \hat{h}}{\partial Z}\right)_{\tau} \mathrm{d} Z
$$

Applying (4.4) to the left side of (4.1) yields

$$
\begin{aligned}
& \left(\frac{\partial h^{2}}{\partial \tau}\right)_{z}=\left(\frac{\partial h^{2}}{\partial \tau}\right)_{Z}+\left(\frac{\partial h^{2}}{\partial Z}\right)_{\tau}\left(\frac{\partial Z}{\partial \tau}\right)_{z}: \\
& \left(\frac{\partial Z}{\partial \tau}\right)_{z}=-\frac{z}{\mathcal{L}^{2}} \frac{\mathrm{d} L}{\mathrm{~d} \tau}=-\frac{Z}{\mathcal{L}} \frac{\mathrm{d} \mathcal{L}}{\mathrm{d} \tau} \Longrightarrow \\
& \left(\frac{\partial h^{2}}{\partial \tau}\right)_{z}=\left(\frac{\partial h^{2}}{\partial \tau}\right)_{Z}-Z\left(\frac{\partial h^{2}}{\partial Z}\right)_{\tau} \frac{1}{\mathcal{L}} \frac{\mathrm{d} \mathcal{L}}{\mathrm{d} \tau}
\end{aligned}
$$

Similarly, applying (4.4) to the right side of (4.1) yields

$$
\begin{aligned}
\left(\frac{\partial^{2}\left(h^{3}\right)}{\partial z^{2}}\right)_{\tau} & =\frac{\partial}{\partial Z}\left(\frac{\partial\left(\hbar^{3}\right)}{\partial Z} \frac{\partial Z}{\partial z}\right)_{\tau}: \\
\left(\frac{\partial Z}{\partial z}\right)_{\tau} & =\frac{1}{\mathcal{L}} \Longrightarrow \\
\left(\frac{\partial^{2}\left(h^{3}\right)}{\partial z^{2}}\right)_{\tau} & =\left(\frac{\partial^{2}\left(\hbar^{3}\right)}{\partial Z^{2}} \frac{1}{\mathcal{L}^{2}}\right)_{\tau} .
\end{aligned}
$$


Substituting (4.7) and (4.10) into (4.1) yields the following governing equation:

$$
\frac{\partial\left(\hbar^{2}\right)}{\partial \tau}=Z \frac{\partial\left(\hbar^{2}\right)}{\partial Z} \frac{1}{\mathcal{L}} \frac{\mathrm{d} \mathcal{L}}{\mathrm{d} \tau}+\frac{2}{3 \mathcal{L}^{2}} \frac{\partial^{2}\left(\hbar^{3}\right)}{\partial Z^{2}} .
$$

Before a difference equation for (4.11) can be obtained, $\mathcal{L}(\tau)$ must be expressed in known terms; the method follows.

The following argument is valid in the limit as $Z \rightarrow 1^{-}$. Expand $h$ in a Taylor series about $Z=1$, where the centering is predicated on satisfying the boundary condition $h=0$ at $Z=1$. Doing so yields

$$
\mathfrak{h}=\sum_{\mathbb{N}} c_{n}(Z-1)^{n}:\left.c_{n} \equiv \frac{\partial^{n} \hbar}{\partial Z^{n}}\right|_{Z=1} .
$$

Substituting (4.12) into (4.11) yields the following weighted expression: ${ }^{9}$

$$
\mathcal{O}(Z-1): \mathcal{L} \frac{\mathrm{d} \mathcal{L}}{\mathrm{d} \tau}=-\frac{2}{3} c_{1}
$$

Notice

$$
\left.c_{1}(\tau) \equiv \frac{\partial \hbar}{\partial Z}\right|_{Z=1} \approx \frac{h(1, \tau)-\hbar(1-\Delta Z, \tau)}{\Delta Z}
$$

\footnotetext{
${ }^{9}$ The $Z$ domain considered is $(\varepsilon, 1)$ for all $\varepsilon \in \mathbb{R}^{+}: \varepsilon \ll 1$, as the following is valid in the limit as $Z \rightarrow 1^{-}$.
} 
yet $f(Z=1, \tau)=0$; thus write (4.14) as

$$
c_{1}(\tau) \approx-\frac{h(1-\Delta Z, \tau)}{\Delta Z} .
$$

Substituting (4.15) into (4.13) and integrating over $[\tau, \tau+\Delta \tau]$ yields

$$
\begin{aligned}
\int_{\mathcal{L}(\tau)}^{\mathcal{L}(\tau+\Delta \tau)} \mathcal{L}^{\prime} \mathrm{d} \mathcal{L}^{\prime} & \approx \frac{2}{3} \int_{\tau}^{\tau+\Delta \tau} \frac{\hbar\left(1-\Delta Z, \tau^{\prime}\right)}{\Delta Z} \mathrm{~d} \tau^{\prime} \Longrightarrow \\
\mathcal{L}^{2}(\tau+\Delta \tau) & \approx \mathcal{L}^{2}(\tau)+\frac{4}{3} \frac{\hbar(1-\Delta Z, \tau)}{\Delta Z} \Delta \tau .
\end{aligned}
$$

Thus an expression for $\mathcal{L}^{2}(\tau+\Delta \tau)$ is known. The time integral was approximated using a left point rule in time. Before continuing observe that the nonlinear $\mathcal{L}$ term in (4.11) can be expressed as

$$
\frac{1}{\mathcal{L}} \frac{\mathrm{d} \mathcal{L}}{\mathrm{d} \tau}=\frac{\mathcal{L}}{\mathcal{L}^{2}} \frac{\mathrm{d} \mathcal{L}}{\mathrm{d} \tau}=\frac{1}{2 \mathcal{L}^{2}} \frac{\mathrm{d} \mathcal{L}^{2}}{\mathrm{~d} \tau} .
$$

It remains to express $\mathrm{d} \mathcal{L}^{2} / \mathrm{d} \tau$ in known terms. Notice (4.17) can be rewritten as

$$
\frac{\mathcal{L}^{2}(\tau+\Delta \tau)-\mathcal{L}^{2}(\tau)}{\Delta \tau}=\frac{4}{3} \frac{\hbar(1-\Delta Z, \tau)}{\Delta Z}
$$

where the left side term is approximately $\mathrm{d} \mathcal{L}^{2} / \mathrm{d} \tau$. The $\mathcal{L}$ terms from (4.11) are now expressed in terms of known quantities; the next step is to create a numerical scheme to solve for $h$. A finite difference scheme for (4.11) is onerous and computationally draining. In order to alleviate numerical stress, introduce a transform that rids the 
nonlinearity of the time derivative in (4.11) as

$$
g \equiv h^{2} .
$$

The transform (4.20) expedites run time and simplifies the difference equation. Applying (4.20) and (4.18) to (4.11) yields

$$
\frac{\partial \mathcal{g}}{\partial \tau}=Z \frac{\partial \mathfrak{g}}{\partial Z} \frac{1}{2 \mathcal{L}^{2}} \frac{\mathrm{d} \mathcal{L}^{2}}{\mathrm{~d} \tau}+\frac{2}{3 L^{2}} \frac{\partial^{2} \mathfrak{g}^{3 / 2}}{\partial Z^{2}} .
$$

Transforming (4.17) and (4.19) with (4.20) respectively yields

$$
\begin{aligned}
\mathcal{L}^{2}(\tau+\Delta \tau) & =\mathcal{L}^{2}(\tau)+\frac{4}{3} \frac{\sqrt{g(1-\Delta Z, \tau)}}{\Delta Z} \Delta \tau \\
\left.\frac{\mathrm{d} \mathcal{L}^{2}}{\mathrm{~d} \tau}\right|_{\tau} & =\frac{4}{3} \frac{\sqrt{g(1-\Delta Z, \tau)}}{\Delta Z} .
\end{aligned}
$$

Before continuing to a finite difference technique for (4.21), a useful claim is stated and proved.

Claim 1. The partial derivative of a function $f$ lifted to the $m \in \mathbb{R}$ power can be finitely differenced lucidly as

$$
\frac{\partial f^{m}}{\partial x}=\frac{\left.f^{m}\right|_{x+\Delta x}-\left.f^{m}\right|_{x}}{\Delta x}+\mathcal{O}(\Delta x)^{2} .
$$


Proof. Consider a typical method for taking a finite difference of $f^{m}$ :

$$
\frac{\partial f^{m}}{\partial x}=\left.m f^{m-1}\right|_{x} \frac{\left.f\right|_{x+\Delta x}-\left.f\right|_{x}}{\Delta x}+\mathcal{O}(\Delta x)^{2}
$$

Expand $\left.f\right|_{x+\Delta x}$ in a Taylor series about $x$ :

$$
\left.f\right|_{x+\Delta x}=\sum_{\mathbb{N}} \frac{\left.\partial_{n} f\right|_{x} \Delta x^{n}}{n !}=\left.f\right|_{x}+\left.\partial_{x} f\right|_{x} \Delta x+\mathcal{O}(\Delta x)^{2} .
$$

Substitute (4.26) through $\mathcal{O}(\Delta x)$ into (4.25) for $\left.f\right|_{x+\Delta x}$, which yields

$$
\begin{aligned}
\frac{\partial f^{m}}{\partial x} & =\left.m f^{m-1}\right|_{x} \frac{\left.f\right|_{x}+\left.\partial_{x} f\right|_{x} \Delta x-\left.f\right|_{x}}{\Delta x}+\mathcal{O}(\Delta x) \\
& =\left.\left.m f^{m-1}\right|_{x} \partial_{x} f\right|_{x}+\mathcal{O}(\Delta x) .
\end{aligned}
$$

Substitute (4.26) through $\mathcal{O}(\Delta x)$ into (4.24) for $\left.f\right|_{x+\Delta x}$, which yields

$$
\begin{aligned}
\frac{\partial f^{m}}{\partial x} & =\frac{\left(\left.f\right|_{x}+\left.\partial_{x} f\right|_{x} \Delta x\right)^{m}-\left.f^{m}\right|_{x}}{\Delta x}+\mathcal{O}(\Delta x) \\
& =\frac{\left.f^{m}\right|_{x}+\left.\left.m f^{m-1}\right|_{x} \partial_{x} f\right|_{x} \Delta x-\left.f^{m}\right|_{x}}{\Delta x}+\mathcal{O}(\Delta x) \\
& =\left.\left.m f^{m-1}\right|_{x} \partial_{x} f\right|_{x}+\mathcal{O}(\Delta x)
\end{aligned}
$$

The equality of (4.28) and (4.31) demonstrates equality in both methods to $\mathcal{O}(\Delta x)$ and thus proves Claim 1. An inductive argument shows this technique holds for the $n^{t h}$ partial derivative.

The validity of Claim 1 implies the forward time, centered space finite difference 
equation for (4.21) is expressed as

$$
\begin{aligned}
& g_{i}^{j+1}=g_{i}^{j}+ \\
& {\left[i\left(g_{i+1}^{j}-g_{i-1}^{j}\right) \frac{1}{4 \mathcal{L}^{j}}\left(\frac{\mathrm{d} \mathcal{L}^{2}}{\mathrm{~d} \tau}\right)^{j}+\frac{2}{3 \mathcal{L}^{j^{2}}} \frac{\mathfrak{g}_{i-1}^{j}{ }^{3 / 2}-2 \mathfrak{g}_{i}^{j / 2}+\mathfrak{g}_{i+1}^{j}{ }^{3 / 2}}{\Delta Z^{2}}\right] \Delta \tau .}
\end{aligned}
$$

where the subscript $i$ is the $i^{\text {th }}$ spatial node and the superscript $j$ is the $j^{\text {th }}$ time node. A finite difference scheme for (4.23) and (4.22) follow:

$$
\mathcal{L}^{j+1}{ }^{2}=\mathcal{L}^{j^{2}}+\frac{4}{3} \frac{\sqrt{g_{n-1}^{j}}}{\Delta Z} \Delta \tau
$$

and

$$
\left(\frac{\mathrm{d}\left(\mathcal{L}^{2}\right)}{\mathrm{d} \tau}\right)^{j}=\frac{4}{3} \frac{\sqrt{\mathfrak{g}_{n-1}^{j}}}{\Delta Z}
$$

where $n$ is the number of spatial nodes predefined by the user. Transforming $h \mapsto g$ from (4.3) yields the following boundary condition:

$$
\mathcal{g}(1,0)=0 .
$$

Then it is clear if some initial condition $h^{1}$ and one more boundary condition, assuming the boundary condition does not not ill-pose (2.10), are applied all subsequent $h$ profiles can be found. Three physical situations are discussed. 


\subsubsection{Capillary Rise (Constant Height)}

As mentioned in Section 3.1.1, the associated boundary condition for the capillary rise regime is $h(0, t)=1$. Notice this boundary condition is invariant under (4.2). Transforming $h \mapsto g$ via (4.20) implies $g(0, \tau)=1$. To satisfy this condition do not finite difference $g_{1}$ since the initial condition (discussed below) implies $g_{1}=1$.

It remains to determine an initial condition. Since no exact solution was found, use either the perturbation technique discussed in Section 3.2.1 or use the variational calculus approach as described in Section 3.3. Since back-transforming a quadratic from $F^{+} \mapsto h$ is straightforward and both approaches show negligible difference in tip behavior, (3.25) through $\mathcal{O}\left(\varepsilon^{7}\right)$ is selected as a proxy for the exact solution. Backtransforming implies (3.25) is equivalently expressed as

$$
h_{a} \equiv h(z, \tau)=0.965616-\frac{0.200595 z}{\sqrt{t}}-\frac{0.0833308 z^{2}}{t}+\mathcal{S}_{2}^{7}
$$

where $\mathcal{S}_{2}^{7}$ denotes the sum of terms from 2 through 7 of the $\eta$ expansion, as illustrated in Figure 3.1. Since $\tau \in \mathbb{R}^{+}$, an initial condition is impossible to obtain. However, letting $\tau=\tau_{0}>0$ implies $h\left(z, \tau_{0}\right)$ is a pseudo initial condition. Then $h\left(z, \tau_{*}\right): \tau^{*} \geq \tau_{0}$

can be found by implementing (4.21). Note the domain for $z$ in (4.36) satisfies both $z \in \mathbb{R}^{+}$and $h(z) \in \mathbb{R}^{+}$. As an addendum, the capillary rise regime conserves volume throughout the entire corner domain (bulk volume plus tip volume). Since $\partial_{z} h \neq 0$ at $z=0$, volume will intrinsically not be constant in the tip, as implied by Appendix B. Then volume is not conserved for this scheme since the control volume is the tip 
only.

To reiterate the code structure, first define the initial condition to be (4.36). Then transform $h \mapsto g$ and run the finite difference scheme (4.32) on all interior space nodes. Reiterate the finite differencing until a user-defined time is reached. Then back-transform $g \mapsto h$. Note the last spatial node is never finitely differenced, as this satisfies (2.11). A MATLAB code for this numerical regime is in Appendix E.

Table 4.1 tabulates numerical results from (4.21) subject to the boundary and initial conditions already described. It is clear the numerical scheme is reliable, as the analytic $\mathcal{C}_{h}$ defined in (3.8) and the numerically calculated $\mathcal{C}_{h}$ converge to the same value (hence $\% \mathcal{C} \rightarrow 0$ ) as the number of space nodes $n$ increases. Additionally, the measure of fit ${ }^{10} \chi$ tends to zero as $n \rightarrow \infty$, which implies the numeric solution $h_{\aleph}$ converges to the analytic solution $h_{a}$. While oscillating slightly, $\chi$ remains relatively small. Figure 4.2 plots time traces in the $h-z$ plane, demonstrating accurate fits at various times. While the multiple time plots undershoot their analytical counterparts, specifically at $z=\mathcal{L}$, Figure 4.1 demonstrates the accuracy achieved when $n$ is relatively large, yielding a $0.4308 \%$ tip maximal error for $n=700$. Figure 4.3 plots the tip length $\mathcal{L}$ through time, which is plotted alongside a function $f=\tau^{1 / 2}$ to demonstrate $\mathcal{L} \sim \tau^{1 / 2}$, which agrees with (3.8). Note Figure 4.3 only begins to follow $\tau^{1 / 2}$ after sufficient time has elapsed. The numerical $\mathcal{C}$ calculated is predicated on $\mathcal{L}>7$, which implies the first $4 \%$ of $\tau$ is ignored.

\footnotetext{
${ }^{10} \mathrm{~A}$ description of the measure $\chi$ is supplied in Appendix D.
} 


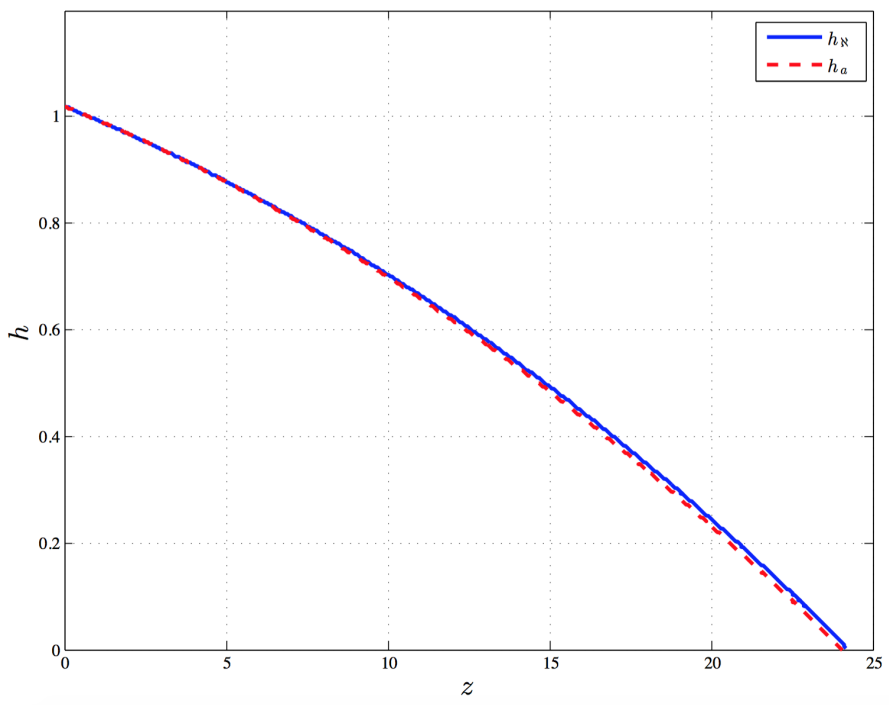

Figure 4.1: Numerical results plotted from the capillary rise regime. The numerically calculated height $h_{\aleph}$ is plotted against $h_{a}$ as defined in (4.36), at time $\tau=100$. The spatial node number is $n=700$.

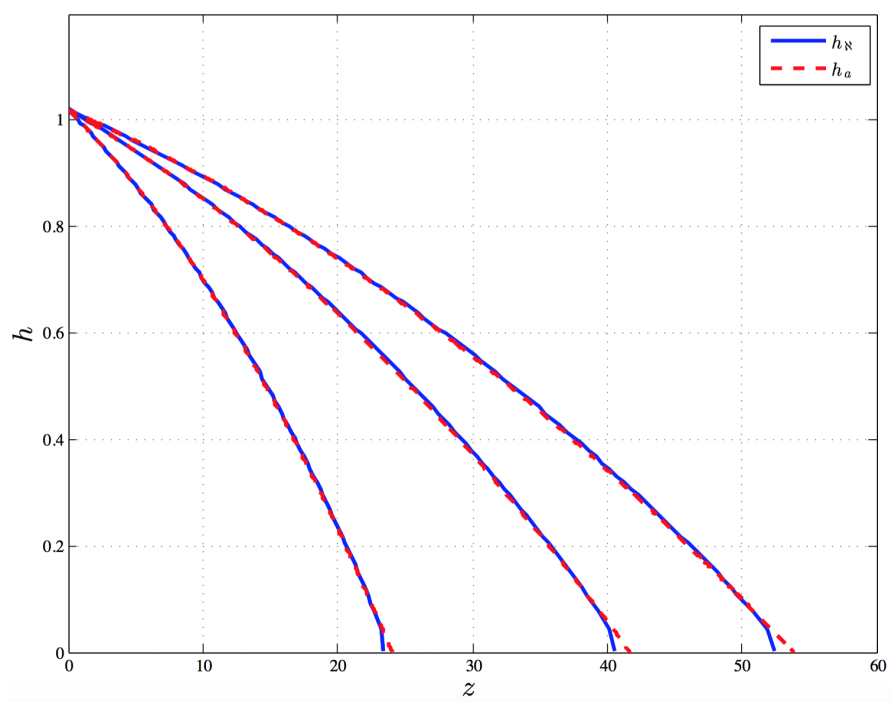

Figure 4.2: Numerical results plotted from the capillary rise regime. The numerically calculated height $h_{\aleph}$ is plotted against $h_{a}$ as defined in (4.36). The spatial node number is $n=150$ and is plotted at times $\tau=100,300$ and 500, where less time corresponds to the left most plot. 


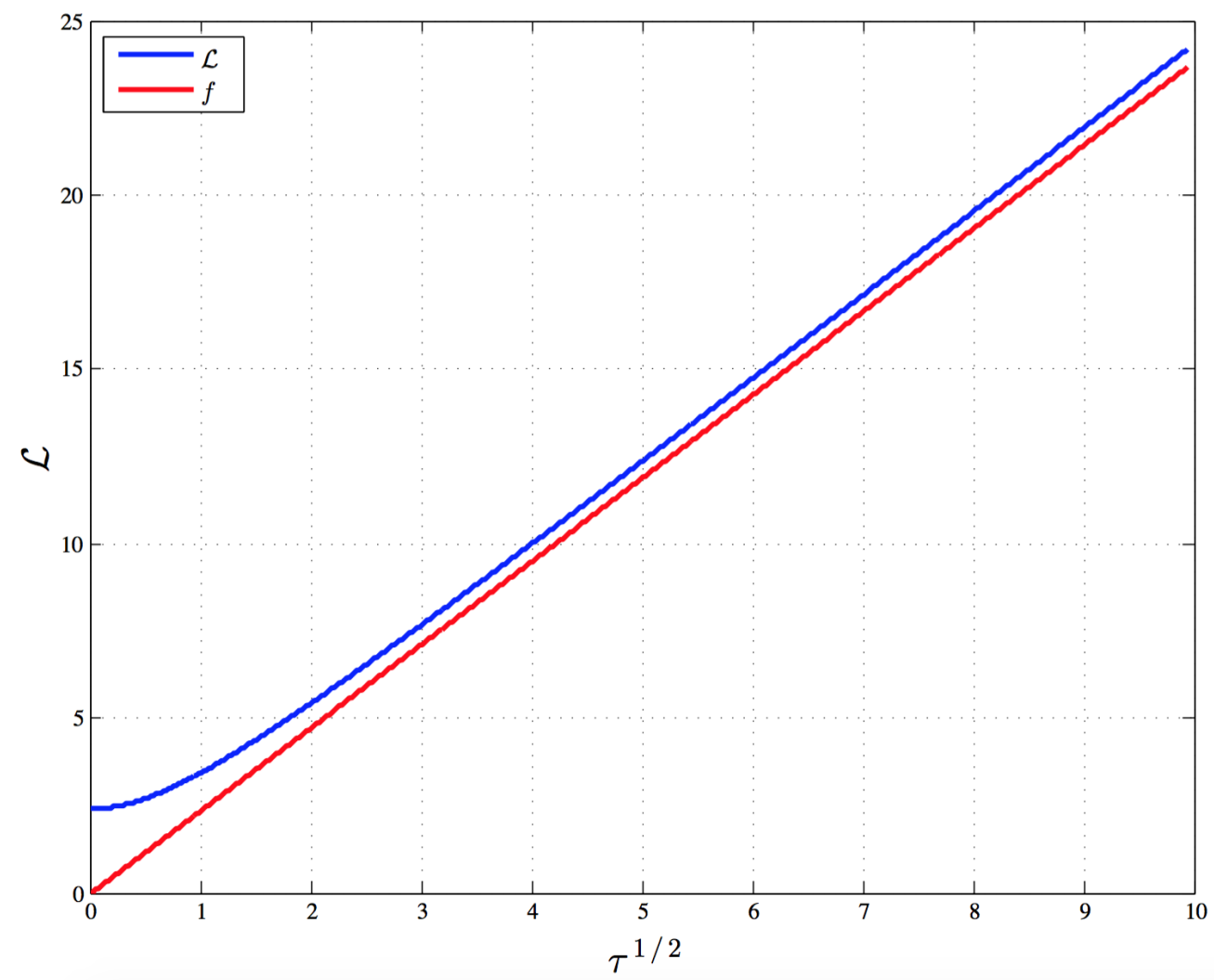

Figure 4.3: Numerical results plotted from the capillary rise regime. The numerically calculated tip $\mathcal{L}$ is plotted against $f=\tau^{1 / 2}$. The spatial node number is $n=700$ and is plotted through times $\tau \in[1,100]$. 
Table 4.1: Capillary rise numerical data collected at $\tau=100$, where $h_{a}$ is defined in (4.36) and $h_{\aleph}$ is the finite differenced numerical solution. $n$ denotes the spatial node number. $\chi$ is defined in (D.1) as a measure of fit. $\% \mathcal{C}$ denotes the percent difference between $\mathcal{C}_{h}$ as defined in (3.8) and $\mathcal{C}_{\aleph}$, where $\mathcal{C}_{\aleph}$ is measured numerically from $h_{\aleph}$ as $\mathcal{L}_{\aleph}=\mathcal{C}_{\aleph} \tau^{2 / 5}$.

\begin{tabular}{|c|c|c|}
\hline$n$ & $\chi$ & $\% \mathcal{C}$ \\
\hline 60 & 0.0508 & 6.1294 \\
\hline 70 & 0.0436 & 5.3886 \\
\hline 80 & 0.0388 & 4.8165 \\
\hline 90 & 0.0344 & 4.3612 \\
\hline 150 & 0.0208 & 2.8370 \\
\hline 300 & 0.0102 & 1.6182 \\
\hline 500 & 0.0016 & 1.1094 \\
\hline 700 & 0.0017 & 0.8869 \\
\hline 1000 & 0.0018 & 0.7177 \\
\hline$\rightarrow \infty$ & $\rightarrow 0$ & $\rightarrow 0$ \\
\hline
\end{tabular}

\subsubsection{Spreading Drop (Constant Volume)}

The spreading drop regime is a symmetric flow about some $z=z_{0} \equiv 0$; then volume is constant for all $z \in \mathbb{R}$. The following boundary condition (to be derived in the upcoming work) $\partial_{z} h=0$ when $z=0$ coupled with Appendix B imply zero fluid flux occurs at $z=0$, and thus volume is constant for $z \in \mathbb{R}^{+}$. We take this fluid as the control volume. Constant volume yields a boundary condition for (4.11); the 
derivation for this boundary condition follows. First rewrite (4.11) as

$$
\mathcal{L} \frac{\partial \hbar^{2}}{\partial \tau}=\left[\frac{\partial\left(Z \hbar^{2}\right)}{\partial Z}-\hbar^{2}\right] \frac{\mathrm{d} \mathcal{L}}{\mathrm{d} \tau}+\frac{2}{3 \mathcal{L}} \frac{\partial^{2} \hbar^{3}}{\partial Z^{2}}
$$

Next move the second term in brackets to the left hand side of the equation, yielding:

$$
\frac{\partial\left(\mathcal{L} h^{2}\right)}{\partial \tau}=\frac{\partial\left(Z h^{2}\right)}{\partial Z} \frac{\mathrm{d} \mathcal{L}}{\mathrm{d} \tau}+\frac{2}{3 \mathcal{L}} \frac{\partial^{2} \hbar^{3}}{\partial Z^{2}}
$$

The total fluid volume over $[0, \mathcal{L}]$ is expressed as $\int_{0}^{\mathcal{L}} \hbar^{2}(z, \tau) \mathrm{d} z$. Transforming $z \mapsto Z$ implies the volume is expressed in the $Z$ system as $\int_{0}^{1} \mathcal{L} h^{2}(Z, \tau) d Z$. Notice now the first term in (4.38) is the time derivative of some infinitesimal volume. Thus integrate (4.38) over $Z \in[0,1]$ to obtain the volumetric time rate of change:

$$
\begin{aligned}
\int_{0}^{1} \frac{\partial\left(\mathcal{L} h^{2}\right)}{\partial \tau} \mathrm{d} Z & =\int_{0}^{1} \frac{\partial\left(Z \hbar^{2}\right)}{\partial Z} \frac{\mathrm{d} \mathcal{L}}{\mathrm{d} \tau} \mathrm{d} Z+\int_{0}^{1} \frac{2}{3 \mathcal{L}} \frac{\partial^{2} \hbar^{3}}{\partial Z^{2}} \mathrm{~d} Z \Longrightarrow \\
\frac{\mathrm{d}}{\mathrm{d} \tau} \int_{0}^{1} \mathcal{L} \hbar^{2} \mathrm{~d} Z & =\left.\frac{\mathrm{d} \mathcal{L}}{\mathrm{d} \tau} Z \hbar^{2}\right|_{0} ^{1}+\left.\frac{2}{3 \mathcal{L}} \frac{\partial\left(\hbar^{3}\right)}{\partial Z}\right|_{0} ^{1} .
\end{aligned}
$$

The commutation of the time derivative under the integral in the left hand side from (4.39) to (4.40) requires $\mathcal{L}$ and $\boldsymbol{h}$ be sufficiently well behaved ${ }^{11}$, which is assumed true by the continuum hypothesis. The right hand side of (4.40) is evaluated via the fundamental theorem of calculus. Conservation of volume requires the left hand side

\footnotetext{
${ }^{11}$ Sufficient behavior assumes $\mathcal{L} h^{2}$ is a compact, finite set and that both $\mathcal{L} h^{2}$ and $\partial_{i} \mathcal{L} h^{2}$ exist continuously [8].
} 
of (4.40) equal zero. It remains to show each term on the right hand side vanishes identically.

The first term on the right hand side of (4.40) is obviously zero at $Z=0$. The first term at $Z=1$ is zero since $f(1, \tau)=0$ (height is zero at the tip). The second term on the right hand side of (4.40) may be evaluated via the chain rule as

$$
\left.\frac{2}{3 \mathcal{L}} \frac{\partial \hbar^{3}}{\partial Z}\right|_{0} ^{1}=\left.\frac{2}{\mathcal{L}} h^{2} \frac{\partial \hbar}{\partial Z}\right|_{0} ^{1}
$$

The boundary condition $h(1, \tau)=0$ implies (4.41) evaluated at $Z=1$ is zero. Then all that remains on the right hand side of $(4.40)$ is $\left.2 \mathcal{L}^{-1} \hbar^{2} \partial_{Z} \hbar\right|_{Z=0}$, which must equal zero to conserve volume. Since $\mathcal{L}$ never equals zero (tip location never retracts) and $h(0, \tau)$ is never zero (conservation of mass), $\left.\partial_{Z} \hat{h}\right|_{Z=0}=\left.0 \Longrightarrow \partial_{Z} \mathfrak{g}\right|_{Z=0}=0$. Thus a second boundary condition for the constant volume regime is

$$
\left.\frac{\partial h}{\partial z}\right|_{z=0}=\left.0 \Longrightarrow \frac{\partial g}{\partial Z}\right|_{Z=0}=0
$$

A finite difference method for obtaining $g_{1}^{j+1}$ follows.

Equation (4.42) implies $g^{3 / 2}(-\Delta Z, \tau)=g^{3 / 2}(\Delta Z, \tau)$. This suggests

$$
g^{3 / 2}(-\Delta Z, \tau)-2 g^{3 / 2}(0, \tau)+g^{3 / 2}(\Delta z, \tau)=2\left[g^{3 / 2}(\Delta z, \tau)-g^{3 / 2}(0, \tau)\right]
$$


So at $Z=0$,

$$
\frac{\mathfrak{g}(0, \tau+\Delta \tau)-\mathfrak{g}(0, \tau)}{\Delta \tau}=\frac{4}{3 \mathcal{L}^{2}} \frac{\left[\mathfrak{g}^{3 / 2}(t,+\Delta z)-g^{3 / 2}(0, \tau)\right]}{(\Delta Z)^{2}} .
$$

Equation (4.44) then implies a finite difference equation for the boundary $Z=0$ is

$$
g_{1}^{j+1}=\frac{4}{3 \mathcal{L}^{2}} \frac{\left[g_{2}^{j 3 / 2}-g_{1}^{j 3 / 2}\right]}{\Delta Z^{2}} \Delta \tau+g_{1}^{j} .
$$

It remains to determine an initial condition. Recognize (3.11) can be transformed from $F^{+} \mapsto h$ as

$$
h_{a} \equiv h(z, \tau)=\frac{3^{2 / 5} 5^{1 / 5}}{2^{9 / 5}}\left(\frac{\epsilon}{F_{A}}\right)^{2 / 5} \tau^{-1 / 5}-\frac{1}{10} z^{2} \tau .
$$

Since $\tau \in \mathbb{R}^{+}$an initial condition is impossible to obtain. However, letting $\tau=\tau_{0}>0$ implies $h\left(z, \tau_{0}\right)$ is a pseudo initial condition. Then $h\left(z, \tau_{*}\right): \tau^{*} \geq \tau_{0}$ can be found by implementing (4.21). Appendix C justifies that any nonzero value for $\epsilon / F_{A}$ does not violate the conservation of volume constraint. However, physically $\epsilon / F_{A}$ is restricted to a "small" positive parameter, as advanced by Weislogel [10]. Note the domain for $z$ in (4.46) satisfies both $z \in \mathbb{R}^{+}$and $h(z) \in \mathbb{R}^{+}$.

To reiterate the code structure, define the initial condition to be (4.46). Then transform $h \mapsto g$ and run the finite difference scheme (4.32) on all interior space nodes. Then run (4.45) on the first space node. Reiterate the finite differencing until 
a user-defined time is reached. Then back-transform $g \mapsto h$. Note the last spatial node is never finitely differenced, as this satisfies (2.11). A MATLAB code for this numerical regime is in Appendix F.

Table 4.2 tabulates numerical results from (4.21) subject to the boundary and initial conditions already described. It is clear the numerical scheme is reliable, as the theoretical $\mathcal{C}_{v}$ defined in (3.11) and the numerically calculated $\mathcal{C}_{v}$ converge to the same value (hence $\% \mathcal{C} \rightarrow 0$ ) as the number of space nodes $n$ increases. Additionally, the measure of fit ${ }^{12} \chi$ tends to zero as $n \rightarrow \infty$, which implies the numeric solution $h_{\aleph}$ converges to the analytic solution $h_{a}$. The spreading drop regime should conserve volume; Table 4.2 demonstrates this, as the percent change in volume $\% \mathcal{V} \rightarrow 0$ as $n \rightarrow \infty$. Figure 4.5 plots time traces in the $h-z$ plane, demonstrating accurate fits at various times. While the multiple time plots undershoot their analytical counterparts, specifically at $z=\mathcal{L}$, Figure 4.4 demonstrates the accuracy achieved when $n$ is relatively large $(n=700)$. Figure 4.6 plots the tip length $\mathcal{L}$ through time, which is plotted alongside a function $f=\tau^{2 / 5}$ to demonstrate numerical agreement with (3.8). The numerical $\mathcal{C}$ calculated is predicated on $\mathcal{L}>2$, which implies the first $3 \%$ of $\tau$ is ignored.

\footnotetext{
${ }^{12} \mathrm{~A}$ description of the measure $\chi$ is supplied in Appendix D.
} 


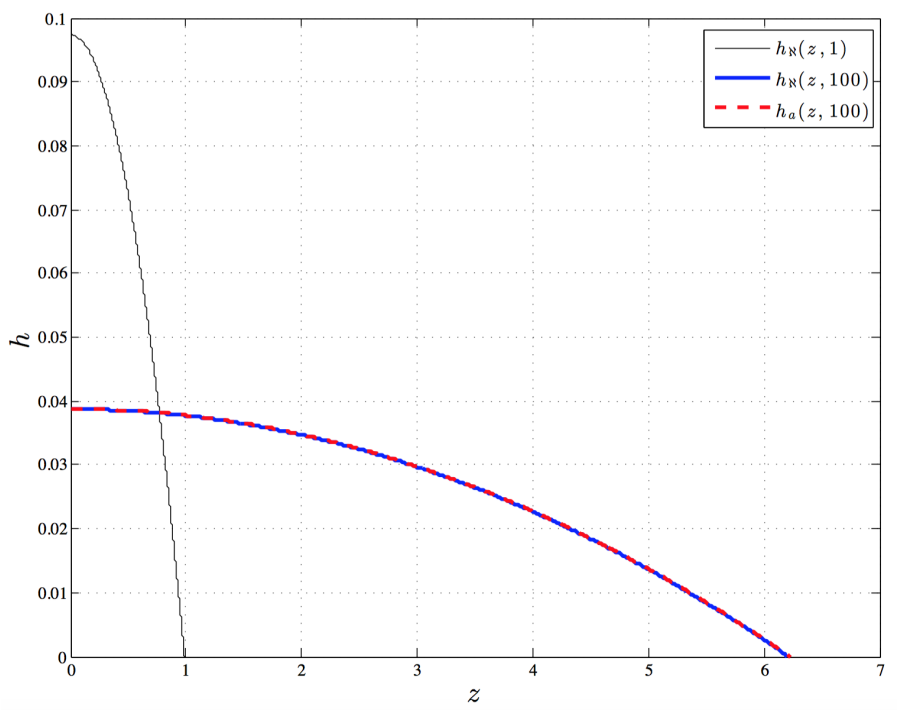

Figure 4.4: Numerical results plotted from the spreading drop regime. The numerically calculated height $h_{\aleph}$ is plotted against (4.36) at time $\tau=100$. The spatial node number is $n=700$ and is plotted at times $\tau=1$ and 100 .

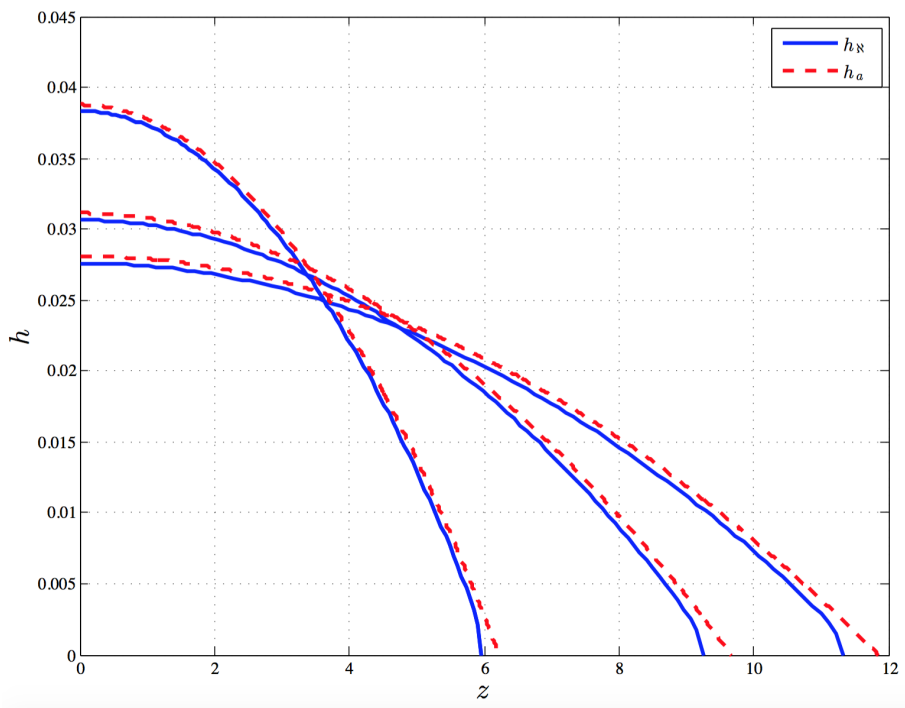

Figure 4.5: Numerical results plotted from the spreading drop regime. The numerically calculated height $h_{\aleph}$ is plotted against $h_{a}$ as defined in (4.46). The spatial node number is $n=100$ and is plotted at times $\tau=100,300$, and 500, where $h\left(0, \tau_{1}\right)<h\left(0, \tau_{2}\right): \tau_{1}<\tau_{2}$. 


\section{Chapter 4}

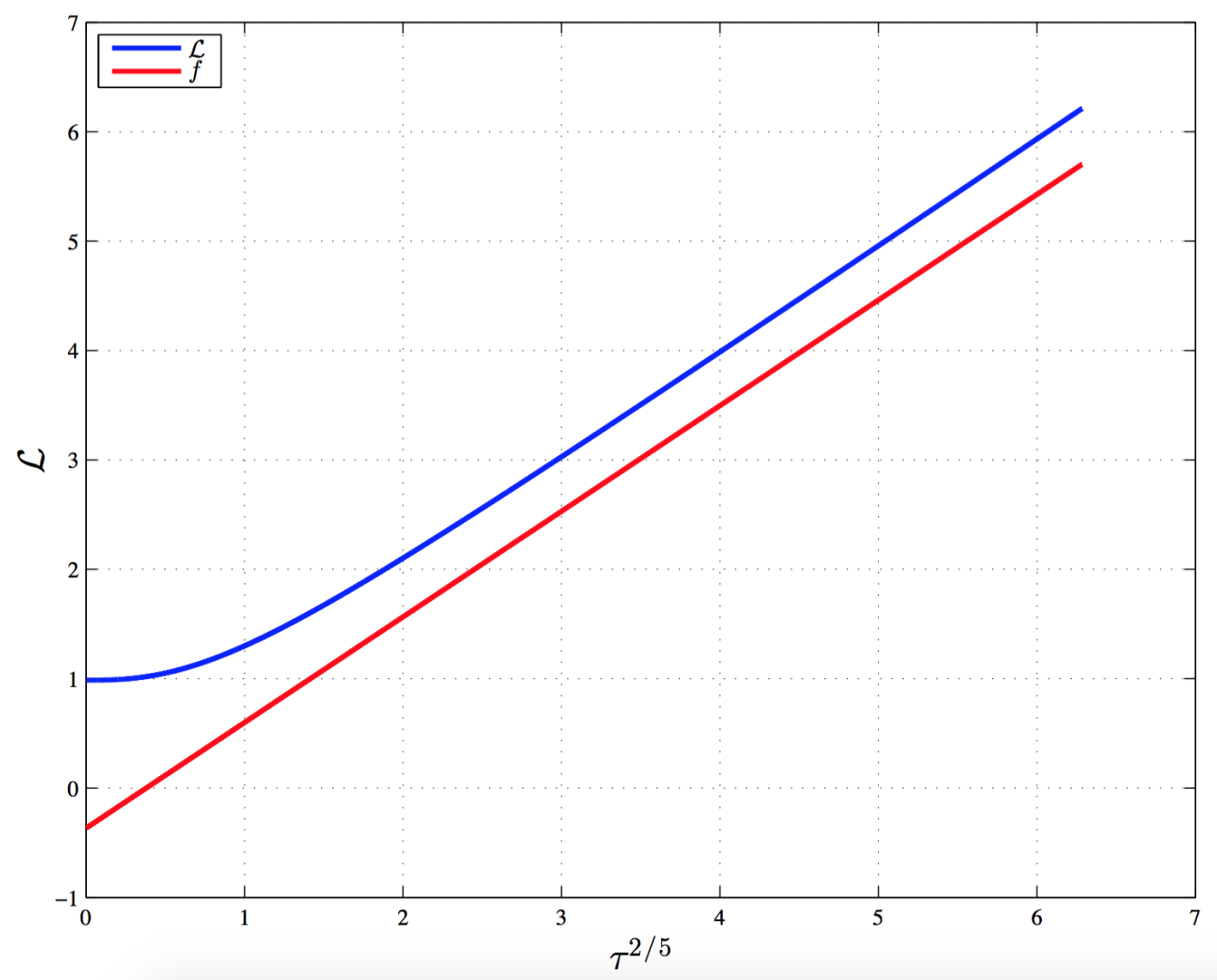

Figure 4.6: Numerical results plotted from the spreading drop regime. The numerically calculated tip $\mathcal{L}$ is plotted against $f=\tau^{2 / 5}$. The spatial node number is $n=700$ and is plotted through times $\tau \in[1,100]$. 
Table 4.2: Spreading drop numerical data collected at $\tau=100$, where $h_{a}$ defined in (4.46) is plotted alongside $h_{\aleph}$, the finite differenced numerical solution. $n$ denotes the spatial node number. $\chi$ is defined in (D.1) as a measure of fit. $\% \mathcal{V}$ denotes the percent volume change between $h_{a}$ and $h_{\aleph} . \% \mathcal{C}$ denotes the percent difference between $\mathcal{C}_{v}$ as defined in (3.12) and $\mathcal{C}_{\aleph}$, where $\mathcal{C}_{\aleph}$ is measured numerically from $h_{\aleph}$ as $\mathcal{L}_{\aleph}=\mathcal{C}_{\aleph} \tau^{2 / 5}$. $\mathcal{C}_{v}$ was calculated with $\epsilon / F_{A}=1 / 100$.

\begin{tabular}{|c|c|c|c|}
\hline$n$ & $\chi$ & $\% \mathcal{V}$ & $\% \mathcal{C}$ \\
\hline 60 & 0.0036 & 6.3065 & 8.7208 \\
\hline 70 & 0.0031 & 5.3746 & 7.8263 \\
\hline 80 & 0.0027 & 4.6772 & 7.1386 \\
\hline 90 & 0.0024 & 4.1368 & 6.5935 \\
\hline 150 & 0.0015 & 2.4294 & 4.7880 \\
\hline 300 & 0.0007 & 1.1872 & 3.3725 \\
\hline 500 & 0.0004 & 0.7044 & 2.7923 \\
\hline 700 & 0.0002656 & 0.5005 & 2.5413 \\
\hline 1000 & 0.0002003 & 0.3489 & 2.3523 \\
\hline$\rightarrow \infty$ & $\rightarrow 0$ & $\rightarrow 0$ & $\rightarrow 0$ \\
\hline
\end{tabular}

\subsubsection{Tapered Corner}

The following analysis attempts to numerically predict the behavior of capillary-driven flow in a tapered corner. This regime emulates the constant height regime but with one caveat: the constant height boundary condition is changed to a bulk meniscus receding linearly in $z$. Figure 1.2 illustrates this flow regime. Due to the complicated nature of this flow, the analysis is divided into two sections. First a physical description of the regime is investigated. Following is a description of the numerical scheme 
used to solve for the height profile. The control volume is defined as the entire fluid in the container, as opposed to simply the wicking tip, as was done in Sections 4.1.1 and 4.1.2.

Physically, fluid in static equilibrium rests in a container with a tapered bottom corner. Gravity is instantaneously "turned off" and fluid begins to wick along the interior edge, as shown in Figure 2.1. The bulk meniscus slowly recedes toward the container corner to balance the mass exchange. As the bulk recedes, its height along each corner decreases in space, denoted $\mathcal{H}_{c}$ in Figures (4.7) and (4.8). The following analysis uses a finite differencing technique to determine the height of the advancing front as a function of time and space. Figures 4.7 and 4.8 illustrate two dimensional analogs of the regime. In fact, since the volume was shown to be a function of $h^{2}$, a two dimensional model is sufficient for illustrative and numerical purposes. Then (2.10) remains the governing PDE for the tip height. Since a finite difference scheme is used, we begin by discretizing a mesh.

Uniformly space the domain elements over the interval $\left[0, \mathcal{L}^{j}\right]$ such that $z_{i}^{j}=$ $(i-1) \Delta z^{j}: i=1,2, \ldots, n$ where $n$ is the total number of space nodes. The subscript $i$ denotes the $i^{t h}$ spatial node and the superscript $j$ denotes the $j^{\text {th }}$ time node. A unitary based indexing is employed; thus $z_{1}^{j}=0$ and $z_{n}^{j}=\mathcal{L}^{j}$. Define the bulk meniscus location $z_{\text {bulk }}^{j} \equiv z_{1}^{j}=0$; thus from an absolute frame of reference the $z$ and $h$ axes move in time. Similarly, define $h_{b u l k}^{j} \equiv h^{j}\left(z_{b u l k}^{j}\right)$. The spacing of each node is defined as $\Delta z^{j} \equiv \mathcal{L}^{j} /(n-1)$. Time nodes are identically defined. Next prescribe an initial 
height, where $h_{i}^{j}$ is bijective to $z_{i}^{j}$. To solve for the new height profile, one complete time step is analyzed, where the employed differencing technique is partitioned into three components.

First define $h^{j}$ as the fluid profile height at some $j^{\text {th }}$ time step. Given $h^{j}$, calculate $\mathcal{V}_{t i p}^{j}$, the tip volume at time $j$. Then transform $h^{j} \mapsto g^{j}$. Finite difference all interior nodes $g_{2}^{j}, \ldots, g_{n-1}^{j}$ according to (4.32). Note $\boldsymbol{g}_{n}^{j}$ is not finitely differenced to satisfy $g(\mathcal{L}, 0)=0$. Since $g_{0}^{j}$ is not in the domain, approximate (4.21) via a forward space, forward time scheme and apply this to $g_{1}^{j}$ (for details on forward space differencing, consult Recktenwald [7]). Then $\mathfrak{g}^{j} \mapsto g^{j+1}$ for all nodes except $g_{1}^{j}$, which is soon discussed. Next back-transform $\boldsymbol{g}^{j+1} \mapsto h^{j+1}$. Note $h$ is transformed from $h_{i}^{j} \mapsto h_{i}^{j+1}$ everywhere except for $h_{b u l k}^{j}$, which has decreased in height but not yet receded in space. Call this temporary bulk height $h_{b u l k}^{j+1 / 2}$.

Secondly, recalculate the temporary new tip volume, $\mathcal{V}_{t i p}^{j+1 / 2}$, which is defined as $h^{j+1}$ for all nodes except the first node, which is $h_{b u l k}^{j+1 / 2}$. It will always be the case that $\mathcal{V}_{t i p}^{j+1 / 2}>\mathcal{V}_{\text {tip }}^{j}$ since volume flows in the $+z$ direction. To conserve mass ${ }^{13}$, the bulk meniscus must lose fluid volume equal to fluid volume the tip gained. Thus $\Delta \mathcal{V}_{\text {tip }}^{j} \equiv \mathcal{V}_{\text {tip }}^{j+1 / 2}-\mathcal{V}_{\text {tip }}^{j}$, the change in tip volume from $j$ to $j+1 / 2$, must equal $\mathcal{V}_{b u l k}^{j}$, the amount of bulk volume to be transferred into the tip region. The following analysis

\footnotetext{
${ }^{13}$ A detailed explanation for the conservation of mass applied to general capillary flow problems is described in Appendix B
} 
demonstrates this volume transfer ${ }^{14}$ :

$$
\Delta \mathcal{V}_{\text {tip }}^{j} \equiv \mathcal{V}_{\text {tip }}^{j+1 / 2}-\mathcal{V}_{\text {tip }}^{j}: \mathcal{V}_{\text {tip }}^{j}=\int_{0}^{\mathcal{L}^{j}} h^{j^{2}} \mathrm{~d} z
$$

Referencing Figure 4.7, $\Delta \mathcal{V}_{\text {tip }}^{j}=\mathcal{V}_{2}-\mathcal{V}_{1}$. As shown in (4.47), a single integral calculates $\Delta \mathcal{V}_{t i p}^{j}$ and is always positive since fluid flows in the $+z$ direction. Since $\Delta \mathcal{V}_{t i p}^{j}=\mathcal{V}_{b u l k}^{j}$, all that remains is to determine $z_{b u l k}^{j+1}$, which is found through $\mathcal{V}_{b u l k}^{j}$. Notice

$$
\begin{aligned}
\mathcal{V}_{\text {bulk }}^{j} & \approx \int_{-z_{\text {bulk }}^{j+1}}^{0} \mathcal{H}_{w}(z)^{2}-\mathcal{H}_{c}(z)^{2} \mathrm{~d} z: \\
\mathcal{H}_{w}(z) & =\frac{H_{w}}{z_{d}}\left(z+z_{d}-\sum_{i=1}^{j} z_{\text {bulk }}^{i}\right) \\
\mathcal{H}_{c}(z) & =\frac{H_{l}}{z_{d}}\left(z+z_{d}-\sum_{i=1}^{j} z_{\text {bulk }}^{i}\right)
\end{aligned}
$$

where $H_{w}$ is the container height at $z_{b u l k}^{1}$ and $H_{l}$ is the linear height recession line at $z_{b u l k}^{1}$. The container depth $z_{d}$ is the distance from the container's corner tip to $z_{\text {bulk }}^{1}$. The container wall is denoted $\mathcal{H}_{w}$ and the linear bulk regression line is denoted $\mathcal{H}_{c}$; both are time-independent, and thus are fixed with respect to the moving coordinate system. The new bulk position $z_{\text {bulk }}^{j+1}$ is now defined by (4.48), and thus (4.50) defines $\mathcal{H}_{c}\left(z_{\text {bulk }}^{j+1}\right) \equiv h_{\text {bulk }}^{j+1}$. Since (4.21) requires $z_{\text {bulk }}^{j}=0$, the $h$ axis must shift from $z_{\text {bulk }}^{j} \mapsto z_{\text {bulk }}^{j+1}$. To shift the $h$ axis from $\mathcal{H}^{j} \mapsto \mathcal{H}^{j+1}$ add $z_{\text {bulk }}^{j+1}$ to each $z_{i}$.

\footnotetext{
${ }^{14}$ The differential $\mathrm{d} z$ in (4.47) and (4.48) is a slight abuse in notation, as $\mathrm{d} z \approx \Delta z=f(j)$. However, since each integral must be discretized to accommodate the discontinuity of $h^{j}$, notation has been relaxed.
} 
Then concatenate the $z$ profile by introducing a new $z_{1} \equiv 0$, thus each $z_{i}^{j} \mapsto z_{i+1}^{j}$. Similarly, concatenate the $h$ profile with $h_{b u l k}^{j+1}$. Notice this expression is the updated height along the constant height boundary. Now there are $n+1$ space nodes, where the spacing is not necessarily uniform due to the additional node. To maintain uniform spacing, define a new set of elements $z_{i}^{*} \in\left[0, \mathcal{L}^{j+1}\right]: \mathcal{L}^{j+1} \equiv \mathcal{L}^{j}+z_{\text {bulk }}^{j+1}$. Define $z_{i}^{*} \equiv(i-1) \Delta z^{*}: i=1,2, \ldots, n$. The new spacing of each node is defined as $\Delta z^{*} \equiv \mathcal{L}^{j+1} /(n-1)$. It remains to determine each new height $h_{i}^{*}$ corresponding to each $z_{i}^{*}$. This is done by a linear interpolation.

Linearly interpolate each $h_{i}^{j+1}$. Call this interpolation function $\mathcal{I}$ and recognize $\mathcal{I}=f(z)$ is a continuous (although not everywhere differentiable) function of $z$ over the domain $\left[0, \mathcal{L}^{j+1}\right]$. Simply define $h_{i}^{*} \equiv \mathcal{I}\left(z_{i}^{*}\right)$. To find the next height at $j+2$ simply reiterate the above procedure, starting by calculating the new volume. 


\section{Chapter 4}

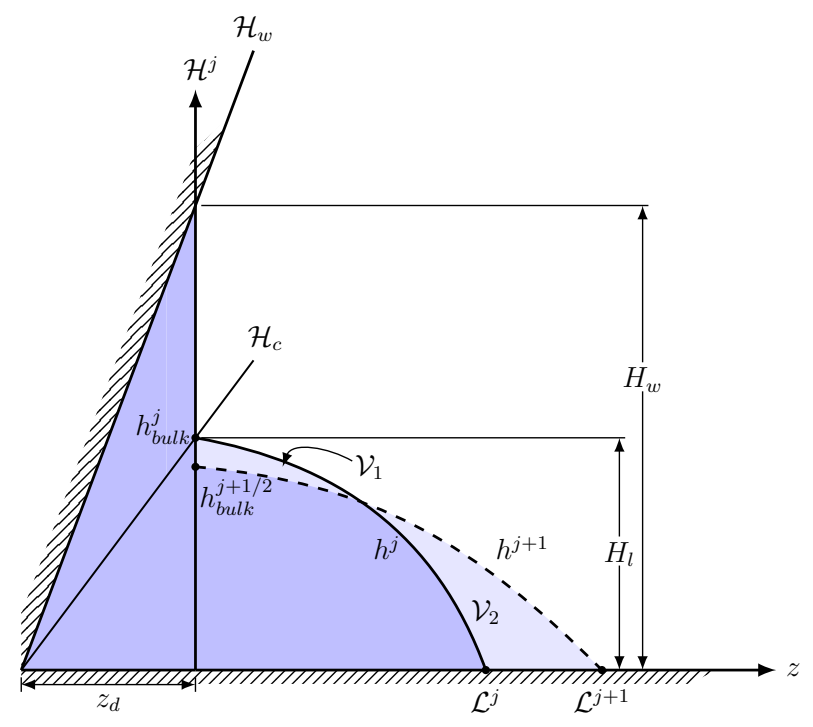

Figure 4.7: The first step in the finite difference scheme of capillary rise in a tapered corner flow. The finite difference approximations (4.32) is conducted on nodes $h_{2}, \ldots, h_{n-1}$, where $h_{1}$ is operated on via a forward space, forward time difference equation analogous to (4.32).

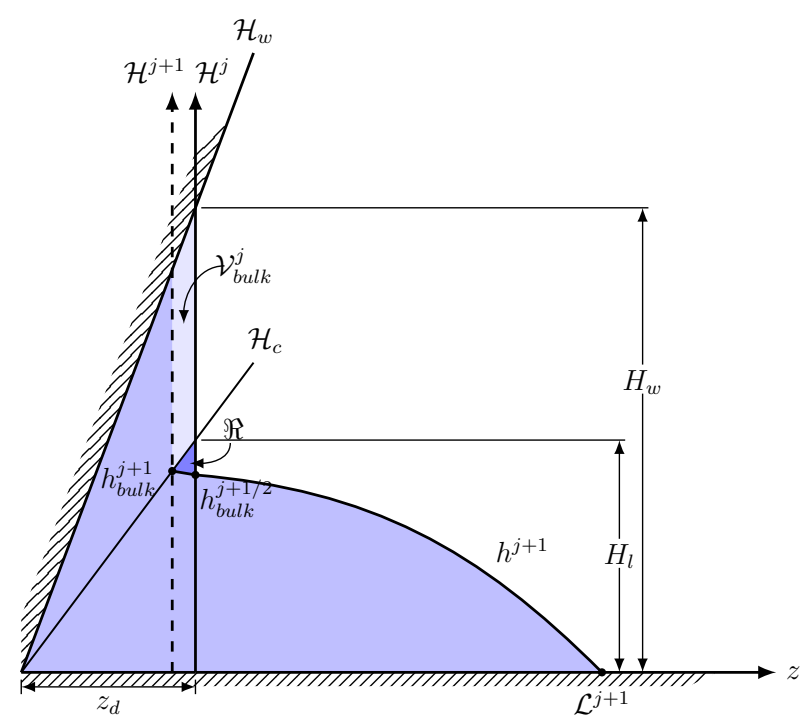

Figure 4.8: The second step in the finite difference scheme of capillary rise in a tapered corner flow. The volume balance described in (4.10) is conducted to find $h_{\text {bulk }}^{j+1}$. 
Before continuing, a brief commentary is needed. Notice (4.10) is not an equality, but an approximation. Equality requires $\Re$ from Figure 4.8 be accounted for. As is, (4.10) omits $\Re$ from the volume balance. Weislogel [12] argues $\Re \ll 1$, and may be ignored when computing a total volume balance. Table 4.3 corroborates this argument, as shown by the percent volume change, which is much less than one. Additionally, $h_{z}(z, \tau)$ should always be negative since fluid always exits the bulk and enters the tip. However, if the time step is sufficiently large with respect to the space step,

it is possible $z_{\text {bulk }}^{j+1}$ could recede sufficiently far along $\mathcal{H}_{c}$ such that $h_{\text {bulk }}^{j+1}<h_{2}^{j+1}$. To circumvent this stability issue, the code is terminated if such an event occurs and a smaller $\Delta \tau$ should be defined.

It remains to determine an initial condition. Since we postulate the tapered corner regime initially emulates the capillary rise regime, define the initial height to be (4.36) with $\tau=1$. To reiterate the procedure, define an initial height. Calculate tip volume. Finite difference all space nodes except the $n^{\text {th }}$. Re-calculate tip volume. Perform a volume balance by subtracting bulk volume equal to the change in tip volume; this uniquely defines an updated $z_{\text {bulk }}$. A space node is added to account for the new bulk position. Re-mesh the grid and re-iterate until a desired time is met. A MATLAB code for this regime is in Appendix G.

Table 4.2 tabulates numerical results from (4.21) subject to the boundary and initial conditions already described. Since both of the difference schemes employed for the spreading drop and capillary rise were shown to be accurate, the tapered corner 
difference technique is believed to be accurate, as all three techniques are predicated on (4.21). Table 4.3 demonstrates the difference technique conserves volume; as the number of space nodes $n$ increases, the change in total volume $\% \mathcal{V}$ tends to zero. Figure 4.9 plots time traces in the $h-z$ plane. Figure 4.10 plots the tip position $\mathcal{L}$ through time, where a log-log scaling is used to compare $\mathcal{L}$ with $\tau^{2 / 5}$ and $\tau^{1 / 2}$.

The conjecture advanced in the abstract hypothesizes the tapered corner's $\mathcal{L}$ initially behaves as the capillary rise regime and then transitions into the spreading drop. This advancement is predicated on the volume per unit area of the corner's cross section. Referencing Figure 2.1, $\partial_{x} \mathscr{V}>0$ where $\mathscr{V}$ is the cross sectional area per unit depth in the $z$ direction; said differently, when moving in the $x$ direction the volume per unit area increases. In fact, the Jacobian of transformation accounts for this when changing from Cartesian to cylindrical (polar) coordinates. This fact suggests the bulk will recede slowly at first to accommodate a large amount of volume, but should increase in time, as there is less volume per unit surface area. The initial behavior then mimics a constant height condition, which corresponds to the capillary rise flow regime, characterized by $\mathcal{L}=\mathcal{O}\left(\tau^{1 / 2}\right)$. As time increases, $h_{b u l k}$, and hence $\mathscr{V}$, decrease. This causes $\partial_{z} h(0, \tau) \rightarrow 0$, which corresponds to the spreading drop boundary condition, characterized by $\mathcal{L} \rightarrow \mathcal{O}\left(\tau^{2 / 5}\right)$. Additionally, increasing $z_{d}$ implies $\partial_{t} \mathscr{V}$ is small, which is to say as time elapses $\mathscr{V}$ will slowly change. A slow change in $\mathscr{V}$ implies height is relatively constant and $h_{\text {bulk }}$ recedes slower than if the change in $\mathscr{V}$ were large. Then for increasing $z_{d}$ we expect $\mathcal{L} \rightarrow \mathcal{O}\left(\tau^{1 / 2}\right)$. Table 4.4 generally corroborates these two conjectures, where $m$ from $\mathcal{L}=\mathcal{C} \tau^{m}$ is tabulated for 
various $H_{w}$ and $z_{d}$ values. Notice as $z_{d}$ and $H_{w}$ increase, $m \rightarrow 1 / 2$. Similarly, as $z_{d}$ and $H_{w}$ decrease, $m \rightarrow 2 / 5$. It is interesting that sufficiently large $z_{d}$ values actually induce $m \rightarrow 0.48 \neq 1 / 2$. Over $99 \%$ of the time is used for the associated $m$ value.

Evidently the behavior for $\mathcal{L}$ initially grows as $\mathcal{L} \sim t^{1 / 2}$ and then transitioning to $\mathcal{L} \sim t^{2 / 5}$ is not justified for all geometries, as Table 4.4 supports early values of $\mathcal{L} \sim t^{2 / 5}$, and thus never behaving as $t^{1 / 2}$. However, regimes which initially adhere to $t^{1 / 2}$ are shown to transition to $t^{2 / 5}$. Figure 4.10 illustrates this, where $\mathcal{L} \sim t^{1 / 2}$ for $t \in[1,3,000]$. Around $t=3,000 \mathcal{L}$ exhibits a growth transition to $t^{2 / 5}$.

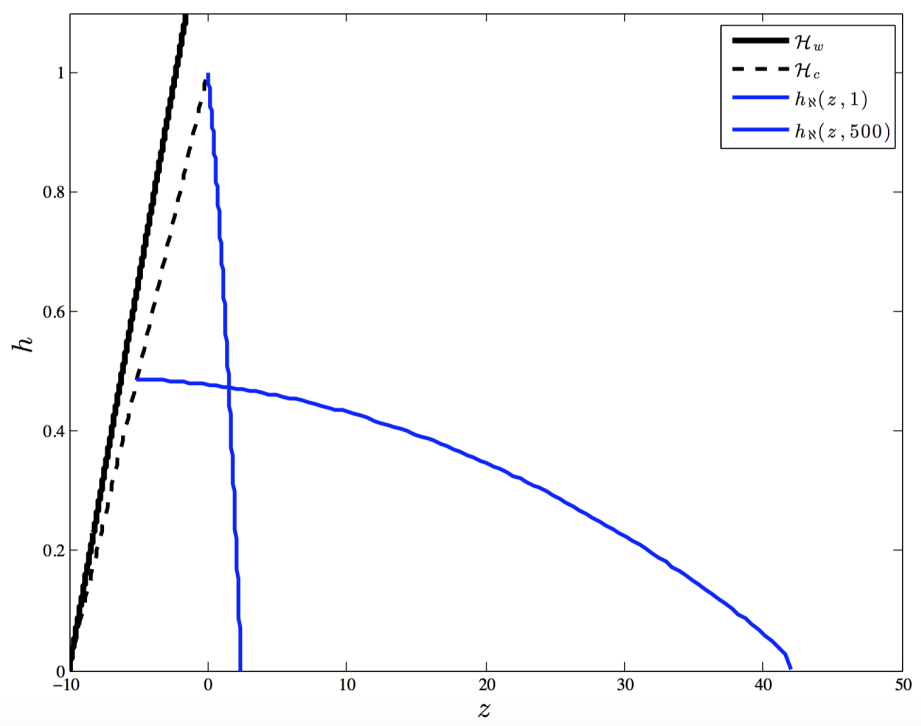

Figure 4.9: Numerical results for the tapered corner regime. The height $h$ is plotted for $n=100$ and is plotted at times $\tau=1$ and 500, where later times exhibit a smaller maximum height. The constant height line $\mathcal{H}_{c}$ is plotted as well as the corner wall $\mathcal{H}_{w}$, which has a maximum height of $H_{w}=1.3$ and $z_{d}=10$. 


\section{Chapter 4}

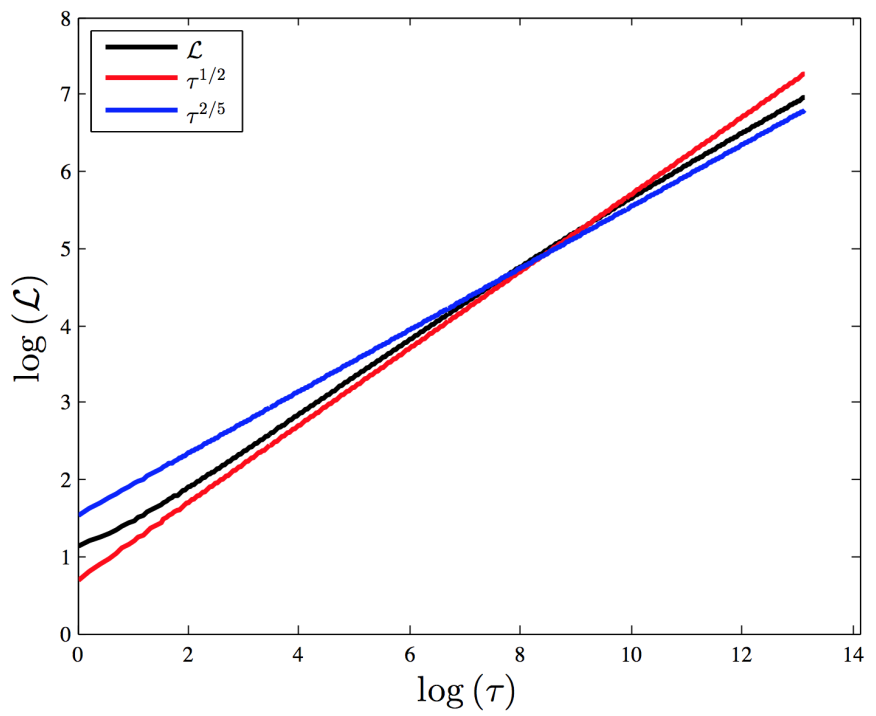

Figure 4.10: Numerical results plotted from the tapered corner regime. The numerically calculated tip $\mathcal{L}$ is plotted against both $\tau^{1 / 2}$ and $\tau^{2 / 5}$ in a log-log plot. The spatial node number is $n=100$ and is plotted for times $1 \leq \tau \leq 500,000$. $\mathcal{H}_{w}=10$ and $z_{d}=1$.

Table 4.3: Tapered corner numerical data collected at $\tau=100$. The parameter $n$ denotes the spatial node number. The slope of the $\log -\log$ plot is denoted $\mathcal{C}_{t}$. The percent change in volume from time $\tau=1$ through $\tau=100$ is denoted $\% \mathcal{V}$. Note $\%$ $\mathcal{V}$ is a total volume calculation, including the bulk volume.

\begin{tabular}{|c|c|}
\hline$n$ & $\% \mathcal{V}$ \\
\hline 60 & 0.0971 \\
\hline 70 & 0.0832 \\
\hline 80 & 0.0726 \\
\hline 90 & 0.0649 \\
\hline 150 & 0.0649 \\
\hline 300 & 0.0391 \\
\hline$\rightarrow \infty$ & $\rightarrow 0$ \\
\hline
\end{tabular}


Table 4.4: Tapered corner numerical data evaluated at $\tau=100$. The spatial node number denoted $n$ is 50 . The slope of the $\log$-log plot $m$ is tabulated for various initial depth lengths $z_{d}$ and various container heights $H_{w}$.

\begin{tabular}{|c|c|c|c|c|c|}
\hline & $H_{w}=1.5$ & $H_{w}=3$ & $H_{w}=5$ & $H_{w}=25$ & $H_{w}=100$ \\
\hline$z_{d}=0.1$ & 0.3923 & 0.3984 & 0.4112 & 0.4719 & 0.4801 \\
\hline$z_{d}=1$ & 0.3997 & 0.4365 & 0.4604 & 0.4798 & 0.4807 \\
\hline$z_{d}=5$ & 0.4277 & 0.4679 & 0.4762 & 0.4806 & 0.4807 \\
\hline$z_{d}=10$ & 0.4463 & 0.4744 & 0.4786 & 0.4807 & 0.4807 \\
\hline$z_{d}=30$ & 0.4687 & 0.4792 & 0.4802 & 0.4807 & 0.4807 \\
\hline$z_{d}=100$ & 0.4796 & 0.4809 & 0.4808 & 0.4807 & 0.4807 \\
\hline$z_{d}=1000$ & 0.4843 & 0.4816 & 0.4811 & 0.4807 & 0.4807 \\
\hline
\end{tabular}

\subsection{Stability}

Stability for (4.32) is measured through the parameter $\Delta \tau / \Delta z^{2}$, the standard stability parameter for a parabolic equation like (2.10). Numerical trials with the aforementioned MATLAB codes show (4.32) is stable when $\Delta \tau / \Delta z^{2} \lesssim 0.6$ for the constant height regime, where as for the spreading drop regime $\Delta \tau / \Delta z^{2} \lesssim 6.0$. Maintaining stability and increasing the final run time without decreasing the number of spatial nodes requires increasing the number of time nodes by some constant multiplier $k$. Doing so reduces $\Delta \tau / \Delta z^{2}$ proportional to $k$. However, a stability criterion is unknown for the tapered corner regime. Changes in $H_{w}$ and $z_{d}$ affect stability, as does decreasing $\Delta z$ so as to avoid $h_{z} \geq 0$. 
Analyzing the stability of time-dependent schemes in non-linear problems is theoretically difficult. Eminent techniques, such as the von Neumann stability procedure, assume the solution to a PDE is separable, which is not qualified for nonlinear equations. Experience has shown that stability is guaranteed for fully implicit schemes (e.g., Backward Euler). Solving the problem implicitly means solving a set of nonlinear equations at each time step. Perhaps the simplest method of solving (4.21) implicitly is to use the method of lines in conjunction with a stiff ODE software package. Such a package transparently solves the set of nonlinear equations at each time step. The method of lines changes the nonlinear PDE into a nonlinear set of coupled ODEs by finite differencing the spatial derivatives, but not the time derivative. So there is an ODE for each grid point. The stiff package automatically integrates these ODEs.

A partially implicit method can be developed for (4.21) that requires a tri-diagonal set of linear equations to be solved at each time step. This can be done using a tridiagonal matrix inversion routine. However, there will be some quantities in the difference equations that would have to be handled explicitly in this approach.

In general, while the above schemes solve (4.21), the primary benefit is decreasing run-time. Since the explicit finite-differenced codes run acceptably fast, an implicit scheme was not applied. However, if several flow regimes were analyzed it would be worthwhile to employ one of the above methods. 


\section{Conclusion}

This body of work describes the governing equations for capillary-driven flows in slender interior corner geometries in zero-gravity environments. The streamwise flow velocity was considered, and after employing a local mass balance a nonlinear partial differential equation governing tip displacement was derived. Three flow regimes were considered: the capillary rise $\mathcal{L} \sim t^{1 / 2}$, spreading drop $\mathcal{L} \sim t^{2 / 5}$, and tapered corner regime, which was conjectured to exhibit $\mathcal{L} \sim t^{1 / 2}$ for small times and then transition to $\mathcal{L} \sim t^{2 / 5}$ for greater times. The former two regimes were well understood by Weislogel [10]. Since no exact solution was known for the capillary rise regime, an original mathematical technique was developed, which perturbed the power of a similarity transform, giving rise to a weighted system of linear ordinary differential equations. The method agreed with both numerical and exact values, exhibiting small errors which exponentially decayed with successive corrective terms.

Once obtaining analytic expressions for the capillary rise and spreading drop regimes, a forward time, centered space, explicit numerical scheme was derived for approximating the governing PDE. A spatial domain transform was introduced to collapse the domain onto time-invariant boundaries. The numerical scheme was shown to converge to analytic values as the number of spatial nodes increased. Since convergence was met and benchmarks set out by Weislogel [10] were satisfied, this scheme was subjected to the third and final regime, the tapered corner. 
A unique code was created for the tapered corner, which applied the domain transformation, included a time-dependent (moving) coordinate system, conserved volume, and satisfied the governing nonlinear partial differential equation with dynamic boundaries. The numerical results for the tapered corner confirm the initial hypothesis this thesis sought to answer; namely that $\mathcal{L}$ initially exhibits tip growth as the capillary rise regime, and after sufficient time elapses, transitions to tip growth of the spreading drop.

\subsection{Recommendations for Future Work}

The development of the perturbational mathematical technique was employed knowing an associated power law parameter $a$. However, it is likely other $a$ values correspond to geometries not here analyzed. An ansatz could be advanced for corresponding $a$ values in such geometries, where analytic solutions could be found for a variety of corner flow conditions. Additionally this technique could be applied to the other physically significant $a$ value regimes, such as $a=1 / 5$, the constant flow regime.

The numerical work can be used to analyze other flow regimes. Since no experiments were conducted, there is much work to be done experimentally. Different tapered geometries can be analyzed and compared to the numerical work herein found. Guesses for $a$ values can be made to try and analytically approximate the tapered corner regime, where likely $a \in(-1 / 5,0)$ if such an parameter is associated with the tapered corner. 


\section{References}

[1] Martin Becker. Principals and Applications of Variational Methods. The MIT Press, 1964.

[2] Daniel A. Bolleddula, Yongkang Chen, Ben Semerjian, Noel Tavan, and Mark M. Weislogel. Compund capillary flows in complex continers: Drop tower test results. Microgravity Science and Technology, June 2010.

[3] Paul Concus and Robert Finn. On the behavior of a capillary surface in a wedge. Proceedings of the National Academy of Sciences, pages 292-299, 1969.

[4] M. Dong and I. Chatzis. The imbibition and flow of a wetting liquid along the corners of a square capillary tube. Journal of Colloid and Interface Science, 172:278-288, January 1995.

[5] F. J. Mayer, J. F. McGrath, and J. W. Steele. A class of similarity solutions for the nonlinear thermal conduction problem. KMS Fusion Incorporated, 16:33933400, 1983.

[6] Enriche Ramé and Mark M. Weislogel. Gravity effects on capillary flows in sharp corners. Physics of Fluids, 21, April 2009.

[7] Gerald Recktenwald. Finite-difference approximations to the heat equation. Unpublished, March 2011.

[8] Michael Spivak. Calculus On Manifolds: A Modern Approach To Classical Theorems Of Advanced Calculus. Westview Press, 1971.

[9] M. M. Weislogel. Capillary flow in interior corners: The infinite column. Physics of Fluids, 13(11), November 2001.

[10] Mark Weislogel. Capillary Flow in an Interior Corner. PhD thesis, Northwestern University, 1996.

[11] Mark M. Weislogel, J. Alex Baker, and Ryan M. Jenson. Quasi-steady capillaritydriven flows in slender containers with interior edges. Journal of Fluid Mechanics, 685:271-305, September 2011.

[12] M.M. Weislogel. Capillary flow in containers of polygonal section. AIAA, 39(12):2320-2326, 2001. 
[13] M.M. Weislogel and S. Lichter. Capillary flow in interior corners. Journal of Fluid Mechanics, 373:349-378, 1998.

[14] Chen Yongkang, Mark M. Weislogel, and Cory L. Nardin. Capillary-driven flows along rounded interior corners. Journal of Fluid Mechanics, 566:235-271, 2006. 
Appendix

\section{Appendix A Determination of $a$ for Spreading Drop}

Assume a mass of fluid volume $H^{\prime 3}$ distributes symmetrically in $z^{\prime}=0$. A volume integral balance shows

$$
H^{\prime 3}=2 \int_{0}^{z_{t i p}^{\prime}} F_{A}^{\prime} h^{\prime 2} \mathrm{~d} z^{\prime}
$$

where $F_{A}^{\prime} h^{\prime 2}$ is the dimensional cross sectional flow area function [10]. Nondimensionalizing (A.1) yields

$$
\begin{aligned}
H^{3} & =2 H^{2} L \int_{0}^{z_{t i p}} F_{A} h^{2} \mathrm{~d} z \Longrightarrow \\
\epsilon & =2 \int_{0}^{z_{t i p}} F_{A} h^{2} \mathrm{~d} z .
\end{aligned}
$$

Applying the change of variables to (A.2) yields

$$
\epsilon=\frac{2 C_{1}^{2} \tau^{2 a}}{C_{2} \tau^{b}} \int_{0}^{\eta_{t i p} / C_{2} \tau^{b}} F_{A} F(\eta)^{2} \mathrm{~d} \eta
$$

From the definition of $\mathcal{L}$ in (3.1) the upper bound of the integral in (A.3) may be re-wrote as

$$
\epsilon=\frac{2 C_{1}^{2} \tau^{2 a}}{C_{2} \tau^{b}} \int_{0}^{\mathcal{L}} F_{A} F(\eta)^{2} \mathrm{~d} \eta .
$$

Equality in (A.4) must be independent of $\tau$, implying $2 a=b$, which, when coupled 


\section{Appendix}

with $b=-(1+a) / 2$ from above yields $a=-1 / 5$ and $b=-2 / 5$. Then (A.4) becomes

$$
1=\frac{2 F_{A} C_{1}^{2}}{\epsilon C_{2}} \int_{0}^{\mathcal{L}} F(\eta)^{2} \mathrm{~d} \eta .
$$

Since $C_{1}$ and $C_{2}$ are arbitrary constants ${ }^{15}$, let $C_{1}=\left(\kappa^{2} / 5\right)^{1 / 5}$ and $\left(C_{2}=5^{2} \kappa\right)^{-1 / 5}$ and recall $\kappa \equiv \epsilon / 2 F_{A}$. Thus (A.5) reduces to

$$
1=\int_{0}^{\mathcal{L}} F(\eta)^{2} \mathrm{~d} \eta .
$$

Additionally, transforming (A.6) with (3.3) yields a solution for $\lambda$ :

$$
\lambda=\left(\int_{0}^{1} F^{+}\left(\eta^{+}\right)^{2} \mathrm{~d} \eta^{+}\right)^{-1 / 5} .
$$

\footnotetext{
${ }^{15} C_{1}$ and $C_{2}$ were generally defined in Chapter 3 . However, for the exotic spreading drop case both constants take different values.
} 
Appendix

\section{Appendix B Volume Integral}

While all three regimes physically require the following mass-integral balance, the finite difference schemes were constructed so as to subvert this requirement (although the spreading drop regime identically satisfies it). The mass balance constraint implies the volumetric flow rate $\dot{Q}$ summed through some time window $\left[t_{i}^{\prime}, t_{f}^{\prime}\right] \equiv \Upsilon^{\prime}$ at some downstream location $z^{\prime}=z_{0}^{\prime}$ must equal the total volume $\mathcal{V}^{\prime}$ over $\left[z_{0}^{\prime}, \mathcal{L}^{\prime}\left(t_{f}^{\prime}\right)\right] \equiv \Omega^{\prime}$ at time $t_{f}^{\prime}$ less the initial volume (if any) prior to $t_{i}^{\prime}$, denoted $\mathcal{V}^{\prime 0}$ over $\Omega^{\prime}$. Note the prime indicates a dimensional quantity. The mathematical expression takes a generalized dimensional form as

$$
\left.\int_{\Upsilon^{\prime}} \dot{Q}^{\prime}\right|_{z_{0}^{\prime}} \mathrm{d} t^{\prime}=\left.\left(\mathcal{V}_{\Omega^{\prime}}^{\prime}-\mathcal{V}_{\Omega^{\prime}}^{\prime \prime}\right)\right|_{t_{f}} .
$$

If $A^{\prime}$ is the dimensional cross sectional surface area orthogonal to the $z$-axis, taking $t_{i}^{\prime}=z_{0}^{\prime}=0,($ B.1) may be expressed via the Reimann integral as

$$
\left.\int_{0}^{t_{f}^{\prime}} \dot{Q}^{\prime}\right|_{z=0} \mathrm{~d} t^{\prime}=\left.\int_{0}^{\mathcal{L}} A^{\prime}\right|_{t=t_{f}} \mathrm{~d} z^{\prime}-\left.\int_{0}^{\mathcal{L}} A^{\prime}\right|_{t=0} \mathrm{~d} z^{\prime} .
$$

Recognize $A^{\prime}=F_{A} h^{2}$ [10]. Substituting this into the right hand side of (B.2) yields

$$
\left.\int_{0}^{\mathcal{L}} A^{\prime}\right|_{t=t_{f}} \mathrm{~d} z^{\prime}=\int_{0}^{\mathcal{L}} F_{A} h^{\prime 2} \mathrm{~d} z^{\prime}
$$

Recognize $\dot{Q}^{\prime}=A^{\prime}\left\langle w^{\prime}\right\rangle \approx A^{\prime}\left\langle w_{o}^{\prime}\right\rangle$ where $w_{o}$ is an $\mathcal{O}(1)$ term in the naive expansion of $w$ in $\epsilon$. Weislogel [10] shows that non-dimensionalizing $\left\langle w_{o}^{\prime}\right\rangle$ and $h_{z}^{\prime}$ implies $\left\langle w_{o}\right\rangle=-F_{i} h_{z}$ 


\section{Appendix}

where $F_{i}$ is a positive geometric function. Then

$$
\begin{aligned}
\left.\int_{0}^{t_{f}} \dot{Q}^{\prime}\right|_{z=0} \mathrm{~d} t^{\prime} & =-\left.\int_{0}^{t_{f}} A^{\prime} F_{i} h_{z}^{\prime}\right|_{z=0} \mathrm{~d} t^{\prime} \\
& =-\left.\int_{0}^{t_{f}} F_{A} F_{i} h^{\prime 2} h_{z}^{\prime}\right|_{z=0} \mathrm{~d} t^{\prime}
\end{aligned}
$$

Substituting (B.3) and (B.5) into (B.2) and nondimensionalizing according to Table 2.1 yields

$$
-\left.F_{i} \int_{0}^{t_{f}} h^{2} h_{z}\right|_{z=0} \mathrm{~d} t=\left.\int_{0}^{\mathcal{L}} h^{2}\right|_{t=t_{f}} \mathrm{~d} z-\left.\int_{0}^{\mathcal{L}} h^{2}\right|_{t=0} \mathrm{~d} z .
$$

Substitute $t=2 \tau / F_{i}$ into (B.6), which yields

$$
-\left.2 \int_{0}^{\tau_{f}} h^{2} h_{z}\right|_{z=0} \mathrm{~d} \tau=\left.\int_{0}^{\mathcal{L}} h^{2}\right|_{\tau=\tau_{f}} \mathrm{~d} z-\left.\int_{0}^{\mathcal{L}} h^{2}\right|_{\tau=0} \mathrm{~d} z .
$$


Appendix

\section{Appendix C Volume Conservation and $\epsilon / F_{A}$}

Consider (2.10) and its exact solution in the spreading drop regime, (3.13), which is restated

$$
h=\frac{1}{2} \lambda^{2} \kappa^{2 / 5}(5 \tau)^{-1 / 5}\left(1-\frac{\eta^{2}}{\lambda^{2}}\right)
$$

where $\eta=\kappa^{-1 / 5} z(5 \tau)^{-2 / 5}$. Substituting this into (C.1) yields

$$
\begin{aligned}
h & =\frac{1}{2} \lambda^{2} \kappa^{2 / 5}(5 \tau)^{-1 / 5}\left(1-\left(\kappa^{-1 / 5} z(5 \tau)^{-2 / 5}\right)^{2} / \lambda^{2}\right) \Longrightarrow \\
& =\frac{1}{2} \lambda^{2} \kappa^{2 / 5}(5 \tau)^{-1 / 5}-\left(\frac{1}{2}(5 \tau)^{-1 / 5}\right)\left(z(5 \tau)^{-2 / 5}\right)^{2} .
\end{aligned}
$$

As an initial condition let $\tau=1$ which implies (C.3) becomes

$$
h=\frac{\lambda^{2} \kappa^{2 / 5}}{2 \cdot 5^{1 / 5}}-\frac{z^{2}}{10}
$$

where $\kappa \equiv \epsilon / 2 F_{A}$. Substituting this into (C.4) yields

$$
h=\frac{\lambda^{2} \epsilon^{2 / 5}}{2^{7 / 5} \cdot 5^{1 / 5} F_{A}^{2 / 5}}-\frac{z^{2}}{10} .
$$

Recall $\epsilon \equiv H / L$. Substituting this into (C.5) yields

$$
h=\Phi-\frac{z^{2}}{10}: \Phi \equiv \frac{\lambda^{2} H^{2 / 5}}{2^{7 / 5} \cdot 5^{1 / 5} F_{A}^{2 / 5} L^{2 / 5}} .
$$


Appendix

A conservation of mass argument in Appendix A implies

$$
\epsilon=2 \int_{0}^{z_{t i p}} F_{A} h^{2} \mathrm{~d} z
$$

Notice $h\left(z_{\text {tip }}\right)=0 \Longrightarrow z_{\text {tip }}=\sqrt{10 \Phi}$, which substitutes into (C.7) as

$$
\epsilon=2 F_{A} \int_{0}^{\sqrt{10 \Phi}} h^{2} \mathrm{~d} z
$$

Substituting (C.6) into (C.8) yields

$$
\begin{aligned}
& \epsilon=2 F_{A} \int_{0}^{\sqrt{10 \Phi}}\left(\Phi-\frac{z^{2}}{10}\right)^{2} \mathrm{~d} z \Longrightarrow \\
& \epsilon=F_{A} \frac{16}{3} \sqrt{\frac{2}{5}} \Phi^{5 / 2} .
\end{aligned}
$$

Substituting the definition of $\Phi$ into (C.10) and $\lambda=(15 / 2)^{1 / 5}$ yields

$$
\begin{aligned}
& \epsilon=F_{A} \frac{16}{3} \sqrt{\frac{2}{5}}\left(\frac{\lambda^{2} H^{2 / 5}}{2^{7 / 5} \cdot 5^{1 / 5} F_{A}^{2 / 5} L^{2 / 5}}\right)^{5 / 2} \Longrightarrow \\
& 1=1 .
\end{aligned}
$$

Equation (C.12) implies (C.1) conserves volume independent of $F_{A}$ and $\epsilon$ at time $\tau=1$, so $F_{A}$ and $\epsilon$ can be arbitrarily chosen as an initial condition. Thus (C.5), which is one valid initial condition and agrees with (4.46), becomes

$$
h=\frac{3^{2 / 5} 5^{1 / 5}}{2^{9 / 5}}\left(\frac{\epsilon}{F_{A}}\right)^{2 / 5}-\frac{z^{2}}{10} .
$$


Appendix

\section{Appendix D Error Measure}

While the tapered corner has no analytic benchmark, the capillary rise and spreading drop regimes do. It is then beneficial to compare the numerical solutions of both to their corresponding analytic solutions. Figures 4.1 and 4.4 plot this comparison for the capillary rise and spreading drop regimes respectively. To determine how well $h_{\aleph}$ approximates $h_{a}$, the following measure $\chi$ is introduced

$$
\chi \equiv \frac{1}{\|\Omega\|} \int_{\Omega}\left|h_{\aleph}-h_{a}\right| \mathrm{d} z
$$

where $\Omega$ is the domain of $h_{a} \in\left[0, \mathbb{R}^{+}\right] \times\left[0, \mathbb{R}^{+}\right]$. Then (D.1) implies $h_{a}$ and $h_{\aleph}$ share the same positive domain. This is not necessarily true. To extend the domain of $h_{\aleph}$, make the following functional extension

$$
h_{\aleph} \equiv \begin{cases}h_{\aleph} & \text { for } z \in[0, \mathcal{L}] \\ 0 & \text { for } z>\mathcal{L} .\end{cases}
$$

The error $\chi$ now accurately measures a magnitude of fit for two continuous functions of a single variable. Note if $\mathcal{E}(z) \equiv\left|h_{\aleph}-h_{a}\right|$ then $\chi$ is simply the average value of $\mathcal{E}$ along $\Omega$. As an addendum, no measure exists definitively stating how "close" any two continuous functions compare over an arbitrary domain. This is in part due to the difficulty of rigorously defining the term "close". 
Appendix

\section{Appendix E MATLAB Code Capillary Rise}

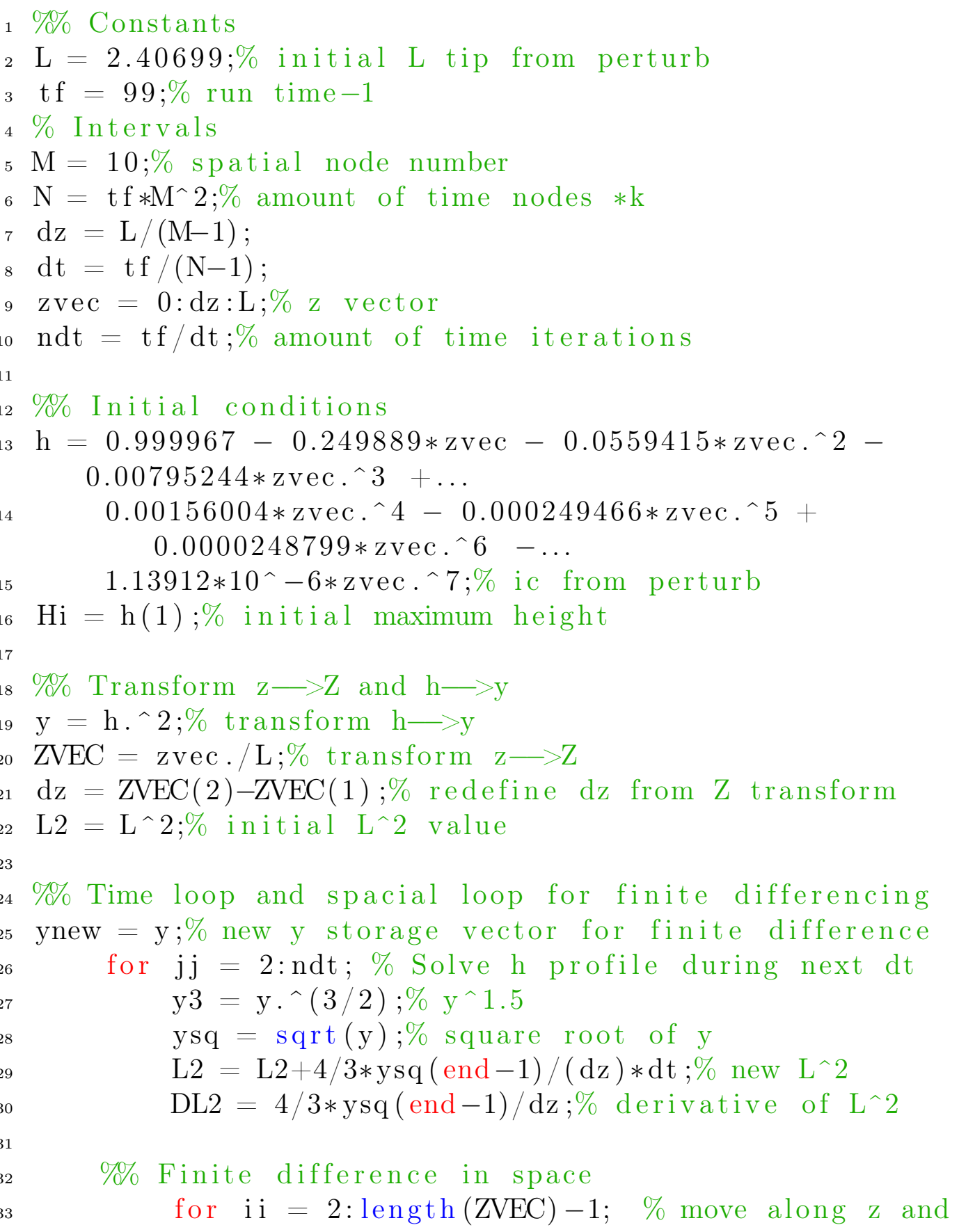




\section{Appendix}

34

35

36

37

38

39

$40 \%$ Transform back $\mathrm{y} \longrightarrow \mathrm{h}$

${ }_{41} \mathrm{~h}=$ ynew.^ $(1 / 2) ; \%$ transform $\mathrm{y} \longrightarrow \mathrm{h}$

$42 \quad \mathrm{zvec}=\mathrm{ZVEC} \cdot * \mathrm{~L}^{\wedge}(1 / 2) ; \%$ transform $\mathrm{Z} \longrightarrow \mathrm{Z}$

$43 \mathrm{dz}=\operatorname{zvec}(2)-\operatorname{zvec}(1) ; \%$ new $\mathrm{dz}$ from $\mathrm{y} \longrightarrow$ h transform 


\section{Appendix}

\section{Appendix F MATLAB Code Spreading Drop}

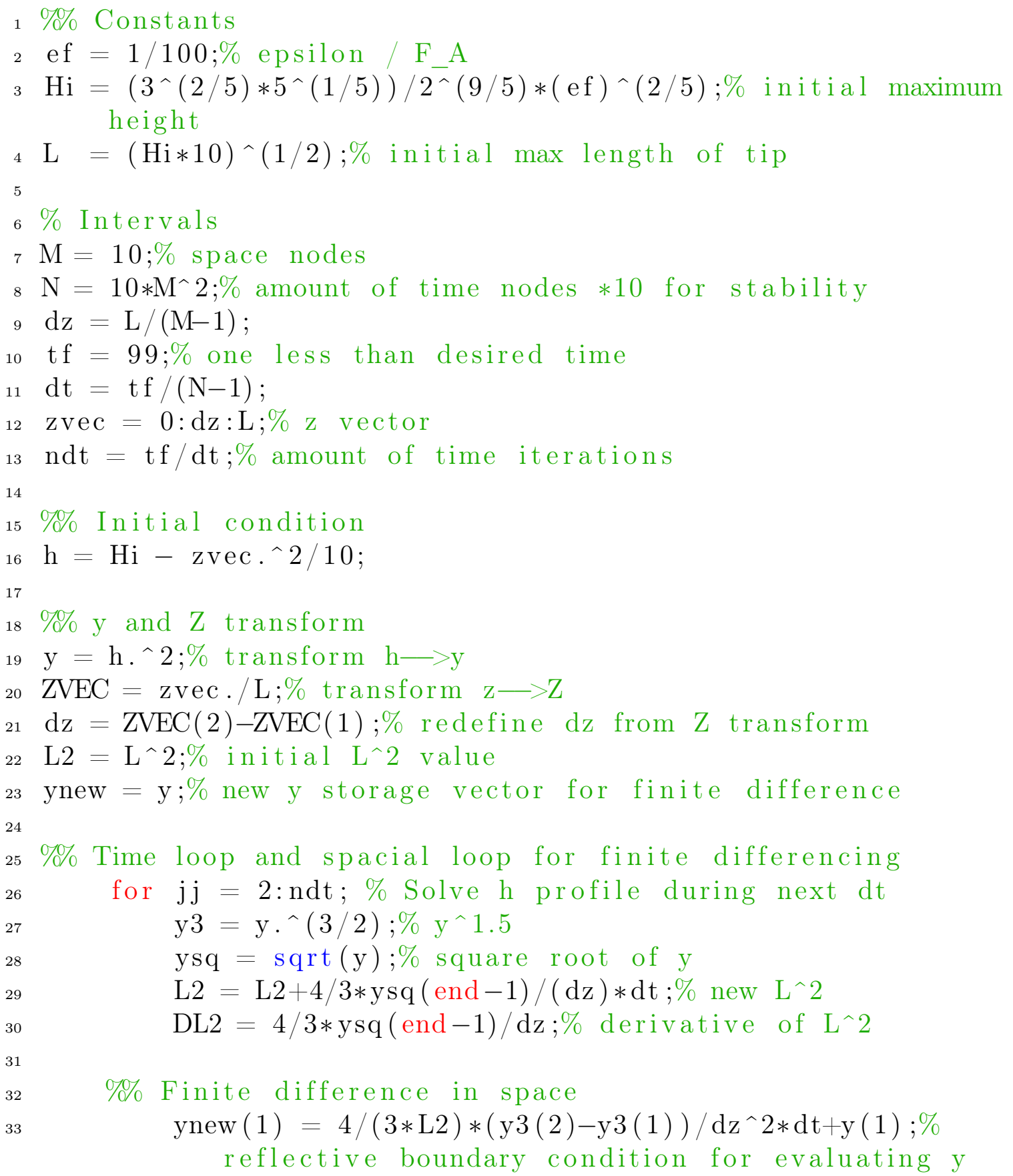




\section{Appendix}

(1)

34

35

36

37

38

39

40

$41 \quad$ end $\%$ for $\mathrm{jj}$

${ }_{42} \mathrm{~h}=$ ynew.^ $(1 / 2) ; \%$ transform $\mathrm{y} \longrightarrow \mathrm{h}$

$43 \quad \mathrm{zvec}=\mathrm{ZVEC} \cdot * \mathrm{~L}^{\wedge}(1 / 2) ; \%$ transform $\mathrm{Z} \longrightarrow \mathrm{Z}$ 
Appendix

\section{Appendix G MATLAB Code Tapered Corner}

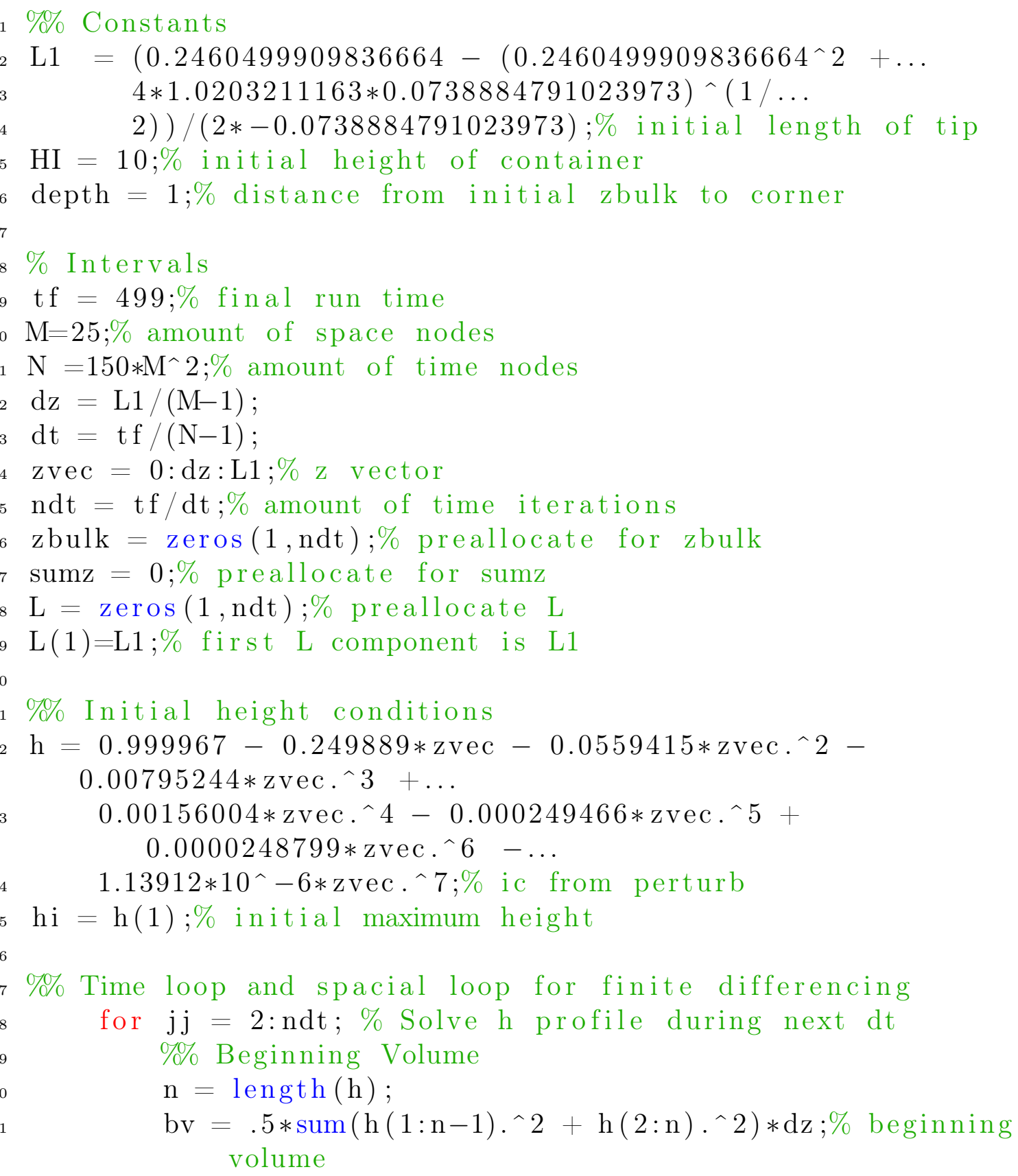




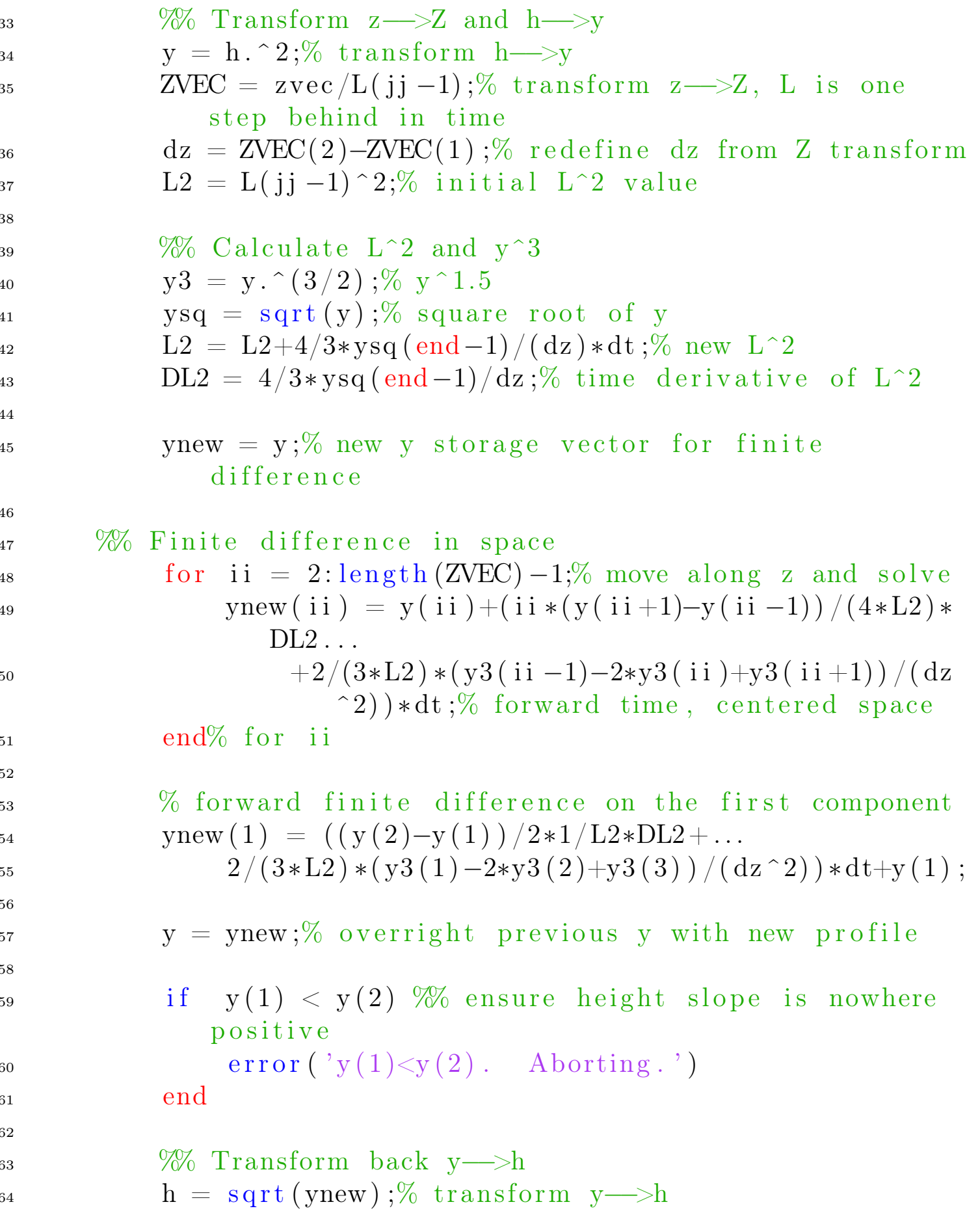




\begin{tabular}{|c|}
\hline $\begin{array}{l}\text { zvec }=\text { ZVEC. } * \text { sqrt }(\text { L } 2) ; \% \text { transform } \mathrm{Z} \longrightarrow \mathrm{z} \\
\mathrm{dz}=\text { zvec }(2)-\mathrm{zvec}(1) ; \% \text { new dz from } \mathrm{y}-\mathrm{h} \text { transform } \\
\mathrm{L}(\mathrm{jj})=\operatorname{sqrt}(\mathrm{L} 2) ; \% \text { define new } \mathrm{L} \text { since previous L } \\
\quad \text { changed }\end{array}$ \\
\hline $\begin{array}{l}\% \% \text { New Volume } \\
\mathrm{nv}=.5 * \operatorname{sum}\left(\mathrm{h}(1: \mathrm{n}-1) \cdot{ }^{\wedge} 2+\mathrm{h}(2: \mathrm{n}) \cdot{ }^{\wedge} 2\right) * \mathrm{dz}\end{array}$ \\
\hline $\begin{array}{l}\% \text { Volume change } \\
\mathrm{dv}=\mathrm{nv}-\mathrm{bv} ; \\
\% \% \text { zbulk, which is cubic so } 3 \text { roots exist } \\
\text { sumz }=\operatorname{sumz}+\text { zbulk }(\mathrm{jj}-1) ; \% \text { sum zbulk } \\
\mathrm{I}=\text { complex }(0,1)\end{array}$ \\
\hline $\begin{array}{l}\text { zbulkc }=1 /(2 *(\mathrm{hi}-\mathrm{HI}) *(\mathrm{hi}+\ldots \\
\mathrm{HI})) *(2 *(\mathrm{hi}-\mathrm{HI}) *(\mathrm{hi}+\mathrm{HI}) *(\mathrm{depth}-\mathrm{sumz})+(1 \\
\quad-\ldots\end{array}$ \\
\hline $\begin{array}{l}\mathrm{I} * \operatorname{sqrt}(3)) *((\mathrm{hi}-\mathrm{HI}) \wedge 2 *(\mathrm{hi}+\ldots \\
\mathrm{HI}) \wedge 2 *(\operatorname{depth} \wedge 3 *(\mathrm{hi}-\mathrm{HI}) *(\mathrm{hi}+\mathrm{HI})+\ldots \\
3 * \operatorname{depth} *(\mathrm{hi}-\mathrm{HI}) *(\mathrm{hi}+\mathrm{HI}) * \mathrm{sumz}^{\wedge} 2+\left(-\mathrm{hi} \wedge 2+\mathrm{HI}^{\wedge} 2\right) \\
\quad * \operatorname{sumz} 3+\ldots\end{array}$ \\
\hline $\begin{array}{l}\left.3 * \operatorname{depth} \wedge 2 *(\mathrm{dv}+(-\mathrm{hi} \wedge 2+\mathrm{HI} \wedge 2) * \operatorname{sumz})))^{\wedge}(1 / 3)\right) \\
\text { zbulk }(\mathrm{jj})=\operatorname{real}(\mathrm{zbulkc}) ; \% \text { zbulk from integral } \\
\quad \text { equation. } \operatorname{Im}(\text { zbulk })<<1\end{array}$ \\
\hline $\begin{array}{l}\% \% \text { Concatenate height and space vector } \\
\text { zvec }=\text { zvec }+ \text { zbulk }(j \mathrm{j}) ; \% \text { shift domain by zbulk } \\
\text { pzvec }=\left[\begin{array}{ll}0 & \text { zvec }] ; \% \text { first spacial point in zvec is z } \\
\quad=0\end{array}\right.\end{array}$ \\
\hline $\begin{array}{l}\mathrm{ph}=[\mathrm{hi} / \operatorname{depth} *(\operatorname{depth}-\mathrm{sumz}) \mathrm{h}] ; \% \text { add new first } \\
\text { height to } \mathrm{h}\end{array}$ \\
\hline $\mathrm{L}(\mathrm{j} \mathrm{j})=\mathrm{L}(\mathrm{j} \mathrm{j})+\mathrm{zbulk}(\mathrm{jj}) ; \%$ update $\mathrm{L}$ with zbulk \\
\hline $\begin{array}{l}\% \% \text { Linearly interpolate and re-mesh the grid } \\
\text { zvec = linspace }(0, \mathrm{~L}(\mathrm{jj}), \mathrm{M}) ; \% \text { new length vector with } \\
\quad \mathrm{M} \text { elements } \operatorname{since} \mathrm{L} \text { has grown } \\
\mathrm{dz}=\text { zvec }(2)-\mathrm{zvec}(1) ; \% \text { new } \mathrm{dz}\end{array}$ \\
\hline
\end{tabular}


Appendix

$\begin{array}{ll}{ }_{95} & \mathrm{~h}(1)=\operatorname{ph}(1) \\ { }_{96} & \mathrm{~h}(\text { end })=\operatorname{ph}(\text { end }) ; \\ { }_{97} & \text { for } \mathrm{i}=2: \mathrm{n}-2 \% \text { remesh grid } \\ & \mathrm{h}(\mathrm{i})=-(\operatorname{ph}(\mathrm{i})-\operatorname{ph}(\mathrm{i}+1)) /(\operatorname{pzvec}(\mathrm{i})-\operatorname{pzvec}(\mathrm{i}+1)) *( \\ & \operatorname{pzvec}(\mathrm{i})-\mathrm{zvec}(\mathrm{i}))+\operatorname{ph}(\mathrm{i}) ; \\ 98 & \text { end\% for i } \\ { }_{99} & \text { end \% for jj }\end{array}$

